\title{
Des compétences aux qualités professionnelles
}

Les repères de la sélection des candidats à l'emploi et leur évaluation

From Skills to Professional Qualities: Job Selection Markers and their Evaluation

\section{Guillemette de Larquier et Emmanuelle Marchal}

\section{(2) OpenEdition}

\section{Journals}

Édition électronique

URL : https://journals.openedition.org/travailemploi/10551

DOI : 10.4000/travailemploi.10551

ISSN : 1775-416X

Éditeur

DARES - Ministère du Travail

Édition imprimée

Date de publication : 15 décembre 2020

Pagination : 5-44

ISSN : 0224-4365

Référence électronique

Guillemette de Larquier et Emmanuelle Marchal, "Des compétences aux qualités professionnelles », Travail et Emploi [En ligne], 163 | 2020, mis en ligne le 01 février 2022, consulté le 26 février 2022. URL : http://journals.openedition.org/travailemploi/10551; DOI : https://doi.org/10.4000/travailemploi. 10551 


\title{
Des compétences aux qualités professionnelles
}

\section{Les repères de la sélection des candidats à l'emploi et leur évaluation"}

\author{
Guillemette de Larquier $^{* *}$, Emmanuelle Marchal ${ }^{* * *}$
}

\begin{abstract}
L'enquête Offre d'emploi et recrutement (Dares, 2016) permet d'analyser les déclarations spontanées des recruteurs pour préciser ce qui détermine la sélection des candidats à l'emploi: de un à trois critères sont cités avec une grande variété de formulations ( $\mathrm{N}=18788$ occurrences). Une analyse de données met en évidence leur agencement d'après les niveaux de qualification. Trois registres de sélection, traduisant des attentes distinctes à l'égard de la main-d'œuvre, sont identifiés : les capacités à s'investir dans son travail, à évoluer dans l'entreprise et à interagir avec autrui. Selon la mobilisation séparée ou conjointe des registres, nous dégageons quatre classes de recrutement, décrites d'après les caractéristiques des entreprises, des emplois et des profils de candidats recrutés. Enfin, nous mettons en évidence les méthodes de recrutement et de sélection associées à ces différentes classes. Les résultats soulignent l'importance des contextes de recrutement dans le choix des qualités valorisées par les recruteurs, et mettent à mal l'idée d'une transversalité des compétences nécessaire à l'occupation des emplois.
\end{abstract}

$\mathrm{L}$ a description des profils de postes dans les annonces, les nomenclatures ou les référentiels de compétence donne à voir la diversité des exigences requises par les employeurs pour occuper des emplois. Les analyses menées en termes de conventions de compétences ont mis en évidence leur rôle dans la sélection à l'emploi, leur mise en forme selon les méthodes et les moyens pour recruter, leur évolution et leurs variations

\footnotetext{
* Nous tenons à remercier Marie-Sophie Dumont sans laquelle ce travail n'aurait pas pu être réalisé. Nous remercions également les participant·es à la journée d'étude «Les enseignements de l'enquête Offre d'emploi et recrutement » organisé par la Direction de l'animation de la recherche, des études et des statistiques (Dares) en novembre 2019, ainsi que les rapporteur-rices anonymes de la revue pour leurs commentaires et conseils avisés. Nous restons seules responsables des erreurs éventuelles du texte.

** Université de Lille, CLERSE (UMR 8019); Centre d'études de l'emploi et du travail (CEET); guillemette.delarquier@univ-lille.fr.

*** CSO Sciences-Po/CNRS; emmanuelle.marchal@sciencespo.fr.
} 
suivant les pays, les caractéristiques des entreprises, des activités ou des contrats (Eymard-Duvernay, Marchal, 1997; Bessy et al., 2001 ; LARQuier, MARCHAL, 2012; RieuCAU, 2015; LARQuier, RieuCau, 2019) ${ }^{1}$. Parmi ces paramètres, la prise en compte des emplois à pourvoir occupe une place importante. L'enquête Offre d'emploi et recrutement de la Direction de l'animation de la recherche, des études et des statistiques (Dares) (Ofer, encadré 1) nous donne l'occasion de creuser cet aspect en partant des formulations foisonnantes adoptées par les recruteurs (plus de 18000 citations recueillies), pour décrire librement le ou les critères (trois au maximum) qui ont joué un rôle décisif dans la sélection des candidats (encadré 2). Leur lecture rapide montre qu'ils ne renvoient pas uniquement à des compétences professionnelles, telles qu'elles sont classiquement repérées dans les curriculum vitae (CV), à partir des cursus de formation ou l'expérience des candidats. Ces critères portent aussi bien sur le dynamisme ou la personnalité du candidat, sa disponibilité ou sa motivation, auxquelles peuvent s'ajouter toutes sortes de considérations d'ordre moral (honnêteté, respect), matériel (posséder une voiture) ou esthétique (bonne présentation). Plus difficiles à légitimer que les compétences professionnelles, ces critères n'en ont pas moins

\section{ENCADRÉ 1}

\section{L'enquête Offre d'emploi et recrutement (Dares, 2016)}

L'enquête Offre d'emploi et recrutement (Ofer) a été menée en 2016 par la Dares auprès de 8510 établissements ayant recruté entre le $1^{\text {er }}$ septembre et le 30 novembre 2015, avec l'objectif de renouveler l'analyse des processus de recrutement déjà réalisée à partir de l'enquête Ofer de 2005 (BERGEAT, RémY, 2017). Il s'agit de la seule enquête au niveau national à décrire précisément les différentes étapes du recrutement, collectant en particulier des informations sur les caractéristiques du poste à pourvoir, les canaux de recrutement, les méthodes de sélection et le profil de la personne embauchée.

Le champ de l'enquête couvre l'ensemble des établissements d'au moins un salarié du secteur concurrentiel non agricole (France métropolitaine et départements d'outre-mer [DOM] ) ayant procédé à un « nouveau » recrutement en CDI ou en CDD de plus d'un mois, sur la période considérée. "Un recrutement est considéré comme "nouveau" dès lors que la personne recrutée n'a pas été embauchée dans le même établissement depuis deux ans et l'a quitté depuis au moins six mois » (BERGEAT, RÉMY, 2017, p. 3).

L'analyse présentée ici exploite les réponses libres à la question QE17 du questionnaire: «Finalement, pour sélectionner le ou les candidats, quels ont été vos principaux critères? » (Saisir en clair. Au maximum trois réponses possibles).

1. En économie des conventions (LARQUIER, 2016), on considère que la compétence des travailleurs n'est pas une donnée naturelle qu'il s'agit juste de communiquer à un employeur potentiel (via des canaux de recrutement efficaces) ou de révéler (grâce à des méthodes de sélection fiables et non biaisées); le recrutement est « un processus de construction des compétences, et non un processus de révélation de celles-ci » (MARCHAL, 2015, p. 15). Ce qu'est un bon candidat relève d'un jugement fondé sur des conventions de compétences plurielles en fonction des emplois, des entreprises, des professions, des activités, etc. 


\section{ENCADRÉ 2}

\section{Les choix opérés à l'occasion du codage des critères}

Partant des 18788 critères cités spontanément par les enquêtés, notre objectif a été de procéder à des regroupements successifs, de façon à améliorer la lisibilité du matériau recueilli et pouvoir le traiter. In fine, 451 repères de sélection ont été identifiés.

1/ La première étape a consisté à opérer un nettoyage du fichier et un regroupement des termes par lemmatisation. Cette étape a été effectuée à l'aide du logiciel OpenRefine dans le but d'éliminer les problèmes de ponctuation, accents, tirets, apostrophes, conjugaisons, pluriels, etc. Elle a conduit à privilégier les majuscules et à opérer des regroupements d'après la proximité morphologique des termes. Cette étape a permis de mettre en évidence l'existence de termes très attractifs utilisés seuls comme MOTIVATION (cité 994 fois), parallèlement à LA MOTIVATION (568), MOTIVATIONS (18).

2/ Lors de la deuxième étape, nous avons traité les expressions comportant plusieurs termes. Le codage a été réalisé à partir des termes fédérateurs contenus dans les expressions en question, en restant au plus près du vocabulaire de l'enquêté, quitte à perdre un peu d'information sur les précisions apportées dans les termes qui suivaient, concernant des qualificatifs, des domaines de connaissance, etc. Deux exemples illustrent cette démarche:

- Autour du terme MOTIVATION, nous avons regroupé des expressions plus précises ou variées comme MOTIVATION POUR LE POSTE, MOTIVATION LORS DE L'ENTRETIEN, LA MOTIVATION A VENIR TRAVAILLER, etc., pour former le repère Motivation qui rassemble 1701 occurrences. Nous l'avons distingué du repère Envie (133 occurrences) qui lui est pourtant proche du point de vue du sens que l'on peut lui accorder : ENVIE DE TRAVAILLER, ENVIE ET VOLONTE D'OCCUPER LE POSTE, ENVIE DE FAIRE LE METIER, L'INTERET POUR LE POSTE ET L'ENVIE DE TRAVAILLER, etc.

- Le repère Connaissance (330 occurrences) regroupe, outre CONNAISSANCE, AVOIR LES CONNAISSANCES REQUISES, CONNAISSANCE DU SECTEUR, CONNAISSANCE DU DOMAINE D'ACTIVITE, DU MILIEU PROFESSIONNEL, DU POSTE DE TRAVAIL, AVOIR UNE CONNAISSANCE SUR LES PLATS ASIATIQUES, etc. Nous l'avons distingué de Connaissance personnelle (113) qui ne renvoie pas à des compétences cognitives mais signale la mobilisation d'un réseau: CONNAISSANCE DU CANDIDAT, DE L'ENTOURAGE, DE LA PERSONNE, DES PARENTS, etc.

Le terme fédérateur privilégié n'est pas nécessairement le premier, mais celui qui est considéré comme le plus informatif. Par exemple, un repère comme Équipe (83 occurrences) n'est jamais utilisé seul. Il fédère différents critères comme LE TRAVAIL EN EQUIPE, SAVOIR FAIRE PARTIE D'UNE EQUIPE, COMPATIBILITE AVEC LES EQUIPES, etc.

Les mots composés sont conservés en l'état (État d'esprit [47 occurrences], Savoir-être [430], Savoir-faire [128], Savoir vivre [11]), ainsi que les expressions dont la fréquence de citation est conséquente (Expérience professionnelle [229], Test situation [35]). 
3/ Plus rarement, et de manière alternative à la démarche du point précédent, quelques repères de sélection ont été construits en procédant à des rapprochements entre synonymes et à des mises en équivalence :

- Le repère Feeling (121 occurrences) regroupe, au-delà du terme FEELING (35) ou LE FEELING (29), et ses déclinaisons mal orthographiées, LE RESSENTI DE LA PERSONNE, L'ALCHIMIE, L'AFFINITE PERSONNELLE, MON RESSENTI, RESSENTI SUR LA FIABILITE DE LA PERSONNE, etc.

- Le repère Localisation (123 occurrences) regroupe des termes comme DISTANCE, DISTANCE DOMICILE/TRAVAIL, DOMICILIATION, LOCALISATION GEOGRAPHIQUE, LIEU DE RESIDENCE, LIEU DE TRAVAIL, LIEU D'HABITATION. Il est distinct du repère Proximité (219) qui figure en tant que tel dans de nombreuses expressions (PROXIMITE AVEC LE DOMICILE, PROXIMITE DU LIEU DE TRAVAIL, PROXIMITE GEOGRAPHIQUE).

une valeur professionnelle dans la mesure où ils sont prisés par les recruteurs. En les rassemblant sous le terme de qualités professionnelles, nous entendons insister sur cette valeur, sans prendre parti sur le fait qu'il s'agisse ou non de compétences. Nous proposons ici d'éclairer la manière dont les recruteurs mobilisent et évaluent ces compétences et qualités, en partant des questions qui affleurent à la lecture de la littérature académique et des rapports institutionnels.

Sans remonter très loin, remarquons que les préoccupations des recruteurs ne se sont jamais cantonnées au strict domaine de «la » qualification ou de « la » compétence. Les préoccupations pour l'apparence, la confiance, la personnalité ou les mœurs des candidats sont déjà très présentes au début du $\mathrm{XX}^{\mathrm{e}}$ siècle et semblent redoubler avec le développement de la population des employés (CHAmBOnNAUd, 1918; KRACAUER, 1929; MiLls, 1970). Les qualités sont contrôlées en recourant à des références et à des recommandations, mais pas seulement. L'histoire des psychotechniques, comme celle de la graphologie, montre que l'on cherche par de nombreux moyens à instrumenter et mesurer toutes sortes de comportements et de traits de personnalité au moment de l'entrée dans les entreprises (EYMARD-DuVERnAY, MARCHAL, 2000; MARCHAL, 2005).

Une littérature abondante s'attache à décrire ces qualités, leur rôle, leurs usages, leurs effets et éventuellement leur développement, sans s'accorder sur la manière de les nommer ou les classer. Il peut être question ${ }^{2}$ de « savoir-être » (BELLIER, 1998), d' « attitudes au travail » (CAPPElli, 1995; CAllaghan, Thompson, 2002 ; Bensidoun, TrancART, 2018), de « compétences sociales » (DÉPRET, FILISETTI, 2001 ; LEDUC, VALLÉRY, 2006), mais aussi de « critères comportementaux » (BOUSSARD-VERRECCHIA, PETRACHI, 2012), de « compétences transversales » (ÉDUCATION PERM ANENTE, 2019a, 2019b) et/ou «transférables » (FRANCE STRATÉGIE, 2017), de « compétences non

2. La diversité des dénominations tient aux préoccupations des auteurs et à leurs appartenances disciplinaires, parmi lesquelles les sciences de l'éducation et de gestion, la psychologie industrielle et la psychologie sociale, la sociologie, l'économie et le droit. 
académiques » (FORMATION EM PLOI, 2015) ou encore de compétences « non cognitives » (HeCKMAN et al., 2006; Algan et al., 2014; KaUtZ et al., 2014). En anglais, l'expression « soft skills» (Moss, Tilly, 1996; ZAMUdio, LiChTER, 2008; Grugulis, VINCENT, 2009; HECKMAN, KAUTZ, 2012) est utilisée pour désigner ce qui ne semble pas ressortir des «hard skills». Les qualités professionnelles sont, en effet, souvent définies par la négative, comme n'étant ni académiques, ni techniques, ni cognitives, mais aucune unanimité ne se dégage véritablement à ce sujet. L'accord ne se fait pas davantage sur leur caractère plus ou moins inné, sur le fait qu'il soit ou non possible d'en faire l'apprentissage, ou s'il faut les considérer comme de vraies compétences. Pour ne pas nous y perdre, notons simplement que ces travaux partent globalement du même constat: ces (nouvelles) qualités importent dans le travail, affectent la productivité et la réussite tant scolaire que professionnelle (CAPPELLI, 1995). Poussant plus loin le raisonnement, des économistes comme James HeckMan et Tim KaUTZ (2012), dont les travaux sont couramment cités, entendent démontrer que les soft skills (et en particulier les traits de personnalité) sont de meilleurs prédicteurs (et la cause) de la réussite (mesurée par le revenu, la santé, le diplôme, etc.) que les compétences cognitives habituellement mesurées par les tests. La prise en compte de ces compétences représenterait donc un enjeu tel pour les employeurs, mais aussi pour les chômeurs qui ne parviennent pas à se faire embaucher, que les politiques publiques en viennent à les valoriser ${ }^{3}$.

L'importance que leur accordent des institutions comme l'Organisation de coopération et de développement économiques (OCDE) s'inscrit dans cette logique. L'enquête PIAAC (Programme for the International Assessment of Adult Competencies) propose ainsi de mesurer et de comparer les «compétences clés » des adultes de 40 pays depuis 2011, en soulignant leur caractère générique et leur utilité pour les employeurs. Outre des compétences cognitives (en calcul, lecture, informatique et langues), on y relève des compétences sociales (qualité de contact, capacité à coopérer, à s'organiser, à apprendre, etc.) ou physiques, et plus récemment des compétences "émotionnelles ${ }^{4} »$. Les institutions européennes ont d'ailleurs précédé le mouvement, en lançant les enquêtes PISA (Program for International Student Assessment) dans le milieu éducatif au tournant des années 2000. Celles-ci insistent sur les compétences «transversales » des élèves ${ }^{5}$, qui affecteraient fortement leur réussite présente et future, par-delà les savoirs académiques (DuRU-BELLAT, 2015). Une telle insistance se retrouve aussi en France, où les publications récentes de France Stratégie (FRANCE

\footnotetext{
3. La prestation de Pôle emploi «Valoriser son image professionnelle » propose ainsi aux demandeurs d'emploi de participer à des ateliers axés sur les savoir-être professionnels (au nombre de 14 d'après la présentation de la méthode) et des mises en situation pratiques. « Vos savoirs et savoir-faire vous ont permis d'obtenir un entretien, votre savoirêtre vous permettra de le réussir » (https://www.pole-emploi.fr/candidat/vos-recherches/preparer-votre-candidature/ valoriser-son-image-pro.html, consulté le 6 décembre 2021).

4. Présentation de l'enquête: http://www1.oecd.org/site/surveyofadultskillspiaac/piaacdesign/, consultée le 6 décembre 2021.

5. Goût des élèves pour le travail en équipe et la compétition, adaptabilité, communication ou capacités d'initiative, etc., font partie des indicateurs retenus.
} 
Stratégie, 2017; Aboubadra-Pauly, Afriat, 2019) et la construction d'un « référentiel partagé des compétences transversales » par Pôle emploi (LAINÉ, 2018; Pôle EMPLOI, 2018) témoignent du regain d'intérêt ${ }^{6}$ manifesté à l'égard de ces compétences, dont la transférabilité entre emplois est soulignée. Ces caractéristiques sont considérées comme des atouts importants pour entretenir l'employabilité des individus, sécuriser les parcours professionnels et favoriser la mobilité sur le marché du travail. De leur côté, les entreprises et les conseils en gestion de ressources humaines ont progressivement introduit des référentiels de « compétences comportementales » déclinés à tous les niveaux de la hiérarchie.

De telles évolutions ne vont pas sans critiques ni dérives, parfois dénoncées devant les tribunaux. De grandes entreprises (comme Airbus, Alcatel, Nestlé, Peugeot) se sont vues ainsi sommées de justifier leur choix d'adopter des critères comportementaux dans leurs référentiels de compétences ${ }^{7}$. La coopération, l'engagement personnel, l'ouverture au changement, le comportement éthique, la prise d'initiative deviennent parfois des indicateurs décisifs dans l'appréciation du personnel. Ces critères s'appliquent-ils uniformément à tous les salariés ou sont-ils ajustés aux fonctions et aux situations de travail ? Peut-on exiger la même chose des cadres et des non-cadres ? Par quels moyens les évaluateurs, comme les évalués, peuvent-ils apprécier si les critères retenus sont remplis? Telles sont quelques-unes des questions que se pose le juge pour apprécier leur pertinence et leur licéité (BousSARD-VERRECCHIA, PETRACHI, 2012). De nombreux travaux critiques relaient les interrogations des juges en insistant sur les paradoxes de l'évaluation, de la rémunération et du développement de ces compétences qui seraient par ailleurs cruciales pour les entreprises. Il en va ainsi du travail non qualifié qui demande de nombreuses qualités et compétences non reconnues comme telles. Celles-ci sont « invisibilisées » par les employeurs, au prétexte qu'elles seraient naturelles (propres aux femmes ou à des catégories ethniques) ou trop difficiles à saisir, entretenant ainsi la dévalorisation du travail (DEMAZIĖRE, MARCHAL, 2018) ${ }^{8}$. Les difficultés d'évaluation coöncident avec le développement des services, dont les tâches et missions (indépendamment du niveau de qualification) ne peuvent être approchées ni avec les conventions de l'industrie (JANY-CATRICE, 2007), ni avec le recul d'une

\footnotetext{
6. Il s'agit bien d'un regain. La présentation du Répertoire opérationnel des métiers et des emplois (ROME) par l'Agence nationale pour l'emploi (ANPE) en 1993 montre que cette préoccupation est déjà présente à ce moment-là et dans sa version précédente (MERLE, 1993).

7. Les plaignants ne sont pas des candidats contestant les conditions de leur évaluation dans le cadre de recrutements (qui prendrait un tel risque?). Ce sont des salariés, et plus souvent des syndicats qui dénoncent les dérives des systèmes d'évaluation du personnel de leur entreprise. Voir par exemple: CA Toulouse, $\mathrm{n}^{\circ}$ 11/00604, 21 septembre 2011; CA de Paris, Pôle 6 ch. 2, no 12/03246, 12 septembre 2013; CA Versailles, nº 16/00669, 2 mai 2017; TGI de Nanterre, $\mathrm{n}^{\circ} 14 / 04685,31$ janvier 2018.

8. Une autre source de critiques a trait à la confusion qui s'instaure entre ces qualités professionnelles et les qualités personnelles des individus, voire leurs caractéristiques intrinsèques. Ce débat ouvre sur la question de la ségrégation et de la discrimination, question particulièrement vive aux États-Unis. Des travaux montrent que la valorisation des soft skills vient nourrir l'exclusion des Noirs américains, y compris lorsqu'ils cherchent à accéder aux emplois peu ou pas qualifiés (Moss, TiLly, 1996). Tous les emplois en effet ne seraient pas uniformément concernés par ces questions. Il s'agit surtout de ceux exposés au regard du public et de clients, pour lesquels les employeurs misent sur les qualités relationnelles et l'apparence (WARHURST, NICKSON, 2007).
} 
certaine régulation sociale fondée sur la logique de qualification (BAILLY, LÉNÉ, 2013). Les obstacles redoublent au moment du recrutement. En effet, les compétences se révèlent au sein des situations de travail; et lorsqu'il s'agit d'analyser ces situations, les compétences de service sont parmi celles les plus difficiles à objectiver au sein de référentiels ou de fiches de poste (CollarD et al., 2015).

Dans cet article, nous proposons de prendre au sérieux cette double question de la modulation des qualités demandées selon les emplois à pourvoir, et celle des moyens mis en œuvre par les entreprises pour les évaluer, en prenant appui sur la richesse de l'enquête Ofer. Cette dernière permet de partir des déclarations spontanées des employeurs ${ }^{9}$ et de traiter ensemble le rôle joué par les compétences traditionnelles (la formation, la qualification et l'expérience) et ces qualités professionnelles sur lesquelles nous venons d'insister, pour comprendre comment s'articule cet ensemble de manière inductive. Cet avantage nous oblige à un travail méthodologique original qui occupera la première partie de l'article. Partant de l'idée que les critères ne sont pas actionnés isolément, que certains s' agrègent et que tous ne sont pas compatibles entre eux, nous allons dégager l'existence de « registres de sélection » fédérant une pluralité de critères en procédant à une analyse factorielle des correspondances. Celle-ci ne sera pas effectuée au hasard mais en partant de l'hypothèse que les repères de sélection se distribuent en fonction des catégories socioprofessionnelles. Procédant ensuite à une classification ascendante hiérarchique, nous mettrons en évidence la cohérence propre des trois registres identifiés et la manière dont ils sont actionnés par les recruteurs, qui peuvent les mobiliser seuls ou en les combinant. Dans la troisième partie, nous caractériserons l'usage de ces registres afin de repérer comment ils sont sollicités selon les emplois, les entreprises et les profils de candidats privilégiés. Nous montrerons enfin, à l'aide de régressions logistiques, que les méthodes de recrutement et de sélection associées à l'usage des registres se distinguent les unes des autres. On n'évalue pas de la même façon un candidat selon que l'accent est mis sur telle ou telle capacité.

\section{La distribution des repères de sélection selon les qualifications}

La diversité des préoccupations des recruteurs se ressent dans la dispersion du vocabulaire mobilisé spontanément pour rendre compte des critères décisifs qui ont présidé à la sélection du candidat recruté. Afin de limiter cette dispersion, nous avons

\footnotetext{
9. Cette démarche se distingue de celle de Frédéric LAINÉ (2016) qui fonde son analyse sur l'exploitation de l'enquête complémentaire à l'enquête Besoins en main-d'œuvre (BMO) 2016, dans laquelle les cinq «principales qualités attendues » sont identifiées a priori : l'expérience, la formation, la motivation/disponibilité, la polyvalence/capacité d'adaptation, la présentation/le relationnel. Dans un travail précédent (LARQUIER, MARCHAL, 2012), nous avons exploité l'édition 2005 de l'enquête $O f e r$ qui soumettait aux enquêtés une liste de 26 critères dont ils déclaraient avoir tenu compte à une étape ou une autre de la sélection. Nous avions alors procédé à un classement distinguant les critères discriminatoires, les capacités physiques, la flexibilité, la personnalité, les qualités de contact, le savoir-faire et la formation.
} 
procédé dans un premier temps à des regroupements d'après la proximité morphologique des expressions utilisées et d'après leur proximité sémantique lorsque cela était nécessaire (encadré 2). Ces regroupements nous ont conduites à nommer 451 repères de sélection. Nous utilisons le terme de repères pour les distinguer des critères spontanément cités par les recruteurs et non codés.

$60 \%$ des 451 repères apparaissent moins de 10 fois, tandis que 34 recouvrent plus de 100 occurrences (annexe 1). En réalité, quatre grands repères de sélection prédominent largement ${ }^{10}$. L'Expérience, la Motivation, la Compétence et la Disponibilité sont cités plus de 1000 fois chacun, voire près de 2000 fois pour l'Expérience ${ }^{11}$. Le repérage de l'expérience apparaît comme une priorité largement partagée à l'occasion d'un recrutement. Cela se vérifie dans la plupart des catégories socioprofessionnelles, sauf pour le recrutement des cadres, marqué d'abord par l'intérêt porté à la Compétence, et celui des ouvriers non qualifiés où domine l'attention portée à la Motivation. Celle-ci semble représenter un moindre enjeu à l'occasion du recrutement des plus qualifiés, tout comme la Disponibilité. Nous lisons ici les premiers indices d'une variation des attentes des recruteurs en fonction du niveau de qualification du poste à pouvoir. Cela motive le recours à une analyse factorielle des correspondances (AFC), pour montrer comment se distribuent les repères de sélection en fonction des emplois regroupés en 6 grandes catégories socioprofessionnelles ou qualifications (encadré 3 ).

Le graphique p. 14 représente le premier plan factoriel issu de l'AFC. Celui-ci donne une très bonne représentation de la dispersion des repères de sélection en reproduisant 72,13\% de l'inertie initiale. C'est la raison pour laquelle nous limitons notre commentaire à la description de ce premier plan qui montre combien l'ordonnancement des repères par les seules catégories socioprofessionnelles est effectif.

L'axe 1, qui reproduit 47,9\% de l'inertie initiale associée au tableau de contingence, ordonne les catégories socioprofessionnelles d'après leur niveau de qualification, avec sur la droite les cadres, puis les professions intermédiaires qui leur sont proches, auxquels s'opposent les ouvriers et employés non qualifiés. De leur côté, les employés qualifiés ne sont pas éloignés du centre. Aux cadres sont associés les termes de Technique ${ }^{12}$ et Compétence, qui laissent présager l'évocation de compétences professionnelles, mais aussi celui de Personnalité qui semble partie prenante dans la définition de ce qu'est un bon cadre. Plus classiquement, on retrouve ensuite Diplôme, Adéquation, Salaire, Manager, Niveau, Potentiel et Formation. Au recrutement du personnel non qualifié sont associés les termes de Ponctualité, Motivation, Présentation, Sourire, Disponibilité, Permis. Contrairement à ce qui se passe pour les cadres, aucun de ces repères ne renvoie stricto sensu à ce que l'on entend habituellement par compétence professionnelle. Il s'agit plutôt de qualités, non perceptibles à la lecture du CV des candidats, qui renvoient à leur attitude au travail.

10. Les quatre repères de sélection les plus cités sont très « purs » et peu travaillés.

11. Tous les termes écrits avec une majuscule renvoient à un repère codé.

12. Nous citons les repères d'après l'importance de leur contribution, de la plus à la moins importante (annexe 2). 


\section{ENCADRÉ 3}

\section{L'analyse factorielle des correspondances et la classification}

Cet encadré méthodologique présente comment nous avons construit les registres de sélection en procédant à une analyse factorielle des correspondances (AFC) et à une classification ascendante hiérarchique $(\mathrm{CAH})$.

Le tableau de contingence soumis à l'AFC croise les 6 catégories socioprofessionnelles (cadre, profession intermédiaire, employé qualifié, employé non qualifié, ouvrier qualifié, ouvrier non qualifié) en colonne avec 224 repères en ligne. Comme indiqué dans l'encadré précédent, 451 repères ont été codés, mais pour éviter que des critères rares contribuent à la construction d'un axe de l'AFC du fait d'une corrélation extrême avec une qualification, ont été supprimés tous les repères qui apparaissaient moins de 6 fois. Le seuil de 6 , qui revient à écarter la moitié des repères, a été choisi car si l'occurrence d'un repère est inférieure à 6 , il y a nécessairement des cases vides dans la ligne correspondante dans le tableau de contingence à 6 colonnes. Sur les 18788 critères cités spontanément par les répondants dans l'enquête Ofer, le tableau analysé contient finalement 18212 «observations », à savoir autant de critères recodés et répartis entre 224 repères de sélection selon la qualification du poste auquel ils étaient associés. En arrière-plan, cela implique 8408 procédures de recrutements sur les 8510 de l'enquête. Les observations ne sont pas pondérées car un même recrutement peut compter jusqu'à 3 fois (puisque jusqu'à 3 critères pouvaient être cités par recrutement). En annexe 1 sont présentées les 34 premières lignes du tableau de contingence.

Les deux premiers axes de l'AFC, interprétés dans le corps du texte, reproduisent $72,13 \%$, autrement dit presque les trois quarts, de l'inertie initiale. Le premier plan factoriel croisant ces deux axes permet donc une bonne représentation de la dispersion des 224 repères selon leur proximité ou leur éloignement dans leur manière d'être associés aux 6 catégories socioprofessionnelles (afin d'être lisibles, seuls apparaissent les 20 repères contribuant le plus à la construction de chaque axe).

L'efficacité de l'AFC à ordonner ainsi de manière lisible les repères à partir des qualifications nous fournit une structure sous-jacente de relations entre les 224 repères. Nous aurions pu juger la proximité ou la distance entre tous les repères en fonction de leur cooccurrence, mais sachant qu'il y a un grand nombre de repères et au mieux 3 repères cités par recrutement, cela aurait abouti à un trop grand nombre de clusters. On choisit donc d'agréger les repères sur la base de l'AFC et de créer ce que nous appelons des registres de sélection à interpréter avec un plus haut niveau de généralité. Ces registres sont issus d'une CAH (mobilisant la distance euclidienne et le critère de Ward) réalisée à partir des coordonnées des repères sur les deux premiers axes de l'AFC qui reproduisent près des trois quarts de l'inertie initiale. L'examen du dendrogramme conduit alors au choix d'une partition en trois classes, soit trois registres (annexe 3).

L'ensemble des traitements a été programmé à l'aide du logiciel $R$ avec, en particulier, les packages FactoMineR, factoextra, cluster, GDAtools et questionr (pour les régressions logistiques ultérieures). 
GRAPHIQUE - Le premier axe factoriel de l'AFC représentant les catégories socioprofessionnelles ( $\triangle$ colonnes de l'analyse) et les repères de sélection

(• lignes de l'analyse) les plus contributifs

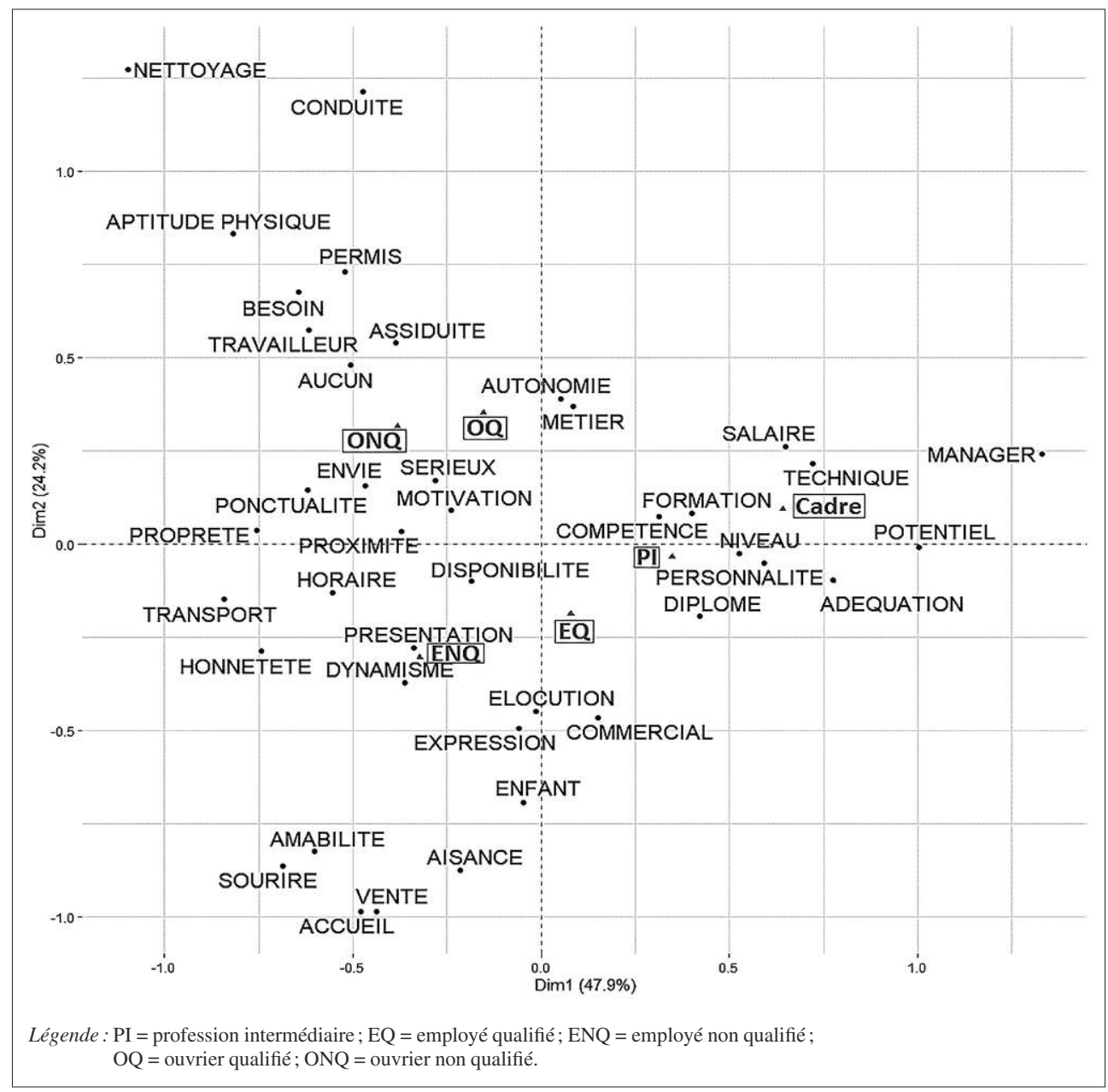

L'axe 2, qui rend compte de $24,2 \%$ de l'inertie initiale, opère une scission entre les emplois d'ouvriers et ceux d'employés, indépendamment de leur niveau de qualification. Aux premiers sont associés les termes de Permis, Conduite, Aptitude physique, Métier, Assiduité et Besoin. La détention d'un permis de conduire, autorisant l'ouvrier à rejoindre une usine ou un chantier, un poste de travail par ses propres moyens, semble ainsi le distinguer d'emblée de l'employé, ainsi que son Aptitude physique à faire son travail et son Assiduité. Pour autant sa qualification n'est pas absente, comme le révèle le terme de Métier dont la possession est valorisée. L'univers sémantique caractérisant la sélection des employés se différencie clairement du précédent: Sourire, Accueil, Présentation, Amabilité, Vente, Dynamisme, Aisance, Élocution, Expression sont corrélés au recrutement d'employés qualifiés ou non. Leur mention 
laisse d'emblée supposer que les candidats seront exposés au regard et aux desiderata de clients ou bénéficiaires auxquels ils proposeront produits et services. En dixième position apparaît enfin la mention du Diplôme.

L'analyse montre ainsi que les priorités accordées aux compétences et aux qualités professionnelles sont modulées selon le niveau du poste à pourvoir, mais pas seulement. La position du cadre n'est pas uniquement déterminée par des compétences professionnelles puisque sa personnalité définit aussi sa valeur. Parallèlement, la possession d'un métier ou d'un diplôme n'est pas négligée à l'occasion de l'embauche des ouvriers ou des employés. Mais les choix sont également guidés par l'attention portée aux conditions de travail et à l'environnement social et matériel dans lequel le candidat est appelé à travailler. En témoignent les repères très différents que mobilisent les recruteurs pour les employés et les ouvriers; on pressent là des diversités sectorielles (LAINÉ, 2016).

La forme triangulaire du graphique dessiné par l'AFC fait également ressortir des différences quant à la diversité des repères que retiennent les employeurs. Ces repères paraissent plus concentrés lorsqu'ils concernent les cadres, donnant à voir l'existence de conventions partagées pour décrire ce qui fait leur valeur professionnelle. La plus grande dispersion des repères choisis pour décrire les emplois d'ouvriers et d'employés non qualifiés souligne a contrario la moindre stabilité des qualités professionnelles qui leur sont, ou non, reconnues. En tout état de cause, quels que soient les types de repères considérés (compétences ou qualités), l'AFC met en lumière que leur choix n'est pas uniforme et accuse des différences marquées selon les qualifications considérées. Ce résultat va à l'encontre du discours portant sur la « transversalité » des qualités requises pour l'occupation de toutes sortes d'emplois, comme si leur possession conditionnait l'employabilité des individus de manière très générale. Cet aspect sera approfondi par la suite.

Pour aller plus loin et comprendre comment s'articulent et sont mobilisés les repères de sélection, nous avons besoin de réduire encore leur dispersion; c'est pourquoi nous allons les agréger en grands registres plus maniables statistiquement. L'hypothèse sous-jacente est que les repères s'excluent mutuellement, ou s'associent au contraire pour former des registres de sélection offrant une certaine cohérence.

\section{S’investir, évoluer, interagir : typologie des registres de sélection}

Tel qu'il se dessine à la suite de la classification ascendante hiérarchique (encadré 3), le regroupement des repères permet d'identifier trois registres de sélection mobilisés séparément ou conjointement ${ }^{13}$, lorsqu'un même recruteur cite trois critères

13. Pour ce qui a trait aux repères, les trois registres sont exclusifs les uns des autres : un même repère n'appartient qu'à un seul registre. En revanche, pour ce qui est des recrutements, ce n'est pas le cas : un même recrutement peut appartenir à un, deux ou trois registres, ce qu'illustre le schéma présenté p. 19. 
bien distincts. Le premier registre regroupe 92 repères de sélection, destinés à cerner la capacité du candidat à s'investir dans son travail, à y être immédiatement opérationnel et efficace; $64,5 \%$ des recrutements de l'enquête y font référence. Le deuxième registre, également riche en qualificatifs ( 83 repères de sélection et $68,6 \%$ des recrutements), est davantage orienté vers la recherche de capacités d'évolution dans la durée au sein de la structure qui recrute. Le troisième registre (49 repères et $19 \%$ des recrutements) concerne centralement les activités au contact de publics ou de clients. Ce registre met l'accent sur la capacité à interagir avec ces derniers et à s'adresser à eux.

Recherche-t-on plutôt un bon travailleur, un bon collaborateur ou quelqu'un disposant d'une certaine aisance relationnelle? Pour faciliter la lecture des repères de sélection qui composent chaque registre, nous allons les cartographier (tableau 1$)^{14}$, en prêtant attention à la manière dont sont qualifiées les compétences d'un côté et les qualités professionnelles de l'autre. Nous mettrons à part ce que nous avons appelé des « indicateurs », qui sont destinés à faciliter le repérage des compétences et qualités. Il peut s'agir d'informations factuelles comme la prise en compte du lieu d'habitation, ou de l'utilisation d'outils de contrôle (réussir un test ou avoir un permis de conduire) jouant un rôle déterminant dans la sélection.

Le registre de la capacité à s'investir dans le travail est orienté vers la recherche d'efficience. Ainsi, du côté des compétences, l'attention se porte sur la maîtrise du Métier, du Savoir-faire, sur la Qualification et le Professionnalisme du candidat. Du côté des qualités valorisées, il s'agit de s'assurer que l'on a affaire à de bons travailleurs. Pour ce faire, l'accent est mis sur le Sérieux, la Rigueur, l'Autonomie, l'Assiduité, le Respect, l'Organisation, l'Efficacité. La Motivation et les termes qui lui sont proches (Envie, Volonté, Engagement ou Implication) font également partie intégrante de ce registre, ainsi que la Disponibilité. Elle doit s'entendre non seulement en termes temporels mais aussi en termes géographiques (Ponctualité, Mobilité, Horaire, Réactivité, Rapidité, Immédiat), comme si l'une et l'autre se conditionnaient mutuellement.

Cette idée est renforcée par la mention d'indicateurs visant à vérifier que le candidat possède bien un Permis de conduire (valable et sans pénalité) s'il doit se déplacer, ou qu'il est domicilié près de son lieu de travail (Proximité, Localisation, Géographique). Pouvoir être là en temps et en heure, y compris pour faire face à des imprévus ou des surcroîts d'activité, fait partie des critères décisifs de ce registre. Dans la mesure où le repérage des bons travailleurs reste difficile à opérer, on remarque parmi les indicateurs l'importance accordée au Feeling, aux Recommandations, aux Connaissances personnelles et aux Références qui viennent appuyer la candidature.

Par rapport au précédent registre, la capacité à évoluer dans l'entreprise est plus souvent qualifiée à l'aide de termes renvoyant à des compétences requises. Ce sont tantôt des compétences générales n'appelant pas de précision particulière (Expérience,

14. Pour décrire chaque registre, nous citons dans ce tableau la moitié des repères de sélection qu'il rassemble, en partant des plus fréquents. Voir l'annexe 3 pour la liste exhaustive de chaque registre. 
TABLEAU 1 - La composition des trois registres de sélection

\begin{tabular}{|c|c|c|}
\hline & Compétences & Qualités \\
\hline \multirow[t]{2}{*}{$\begin{array}{l}\text { Capacité à s'investir } \\
\text { dans le travail }\end{array}$} & $\begin{array}{l}\text { Compétences opérationnelles: } \\
\text { Métier, Savoir-faire, Qualification, } \\
\text { Professionnalisme, Aptitude, Capacité, } \\
\text { Professionnelle. }\end{array}$ & $\begin{array}{l}\text { Bon travailleur: Sérieux, Rigueur, } \\
\text { Comportement, Autonomie, Assiduité, } \\
\text { Confiance, Attitude, Respect, Organisation, } \\
\text { Compréhension, Efficacité, Travailleur, } \\
\text { Aptitude physique. } \\
\text { Disponibilité: Disponibilité, Ponctualité, } \\
\text { Horaire, Mobilité, Réactivité, Rapidité, } \\
\text { Immédiat. } \\
\text { Implication dans le travail: Motivation, } \\
\text { Envie, Volonté, Engagement, Implication. }\end{array}$ \\
\hline & \multicolumn{2}{|c|}{$\begin{array}{l}\text { Indicateurs: Proximité, Permis, Localisation, Feeling, Recommandation, Connaissance } \\
\text { personnelle, Base, Âge, CV, Référence, Géographique, Essai, Besoin. }\end{array}$} \\
\hline \multirow[t]{2}{*}{$\begin{array}{l}\text { Capacité à évoluer } \\
\text { dans l'entreprise }\end{array}$} & $\begin{array}{l}\text { Compétences générales: Expérience, } \\
\text { Compétence, Diplôme, Expérience } \\
\text { professionnelle, Formation, Niveau, Profil, } \\
\text { Parcours, Potentiel, Cursus. } \\
\text { Compétences spécialisées : Connaissance, } \\
\text { Technique, Langue, Expérience } \\
\text { spécialisée, Anglais, Informatique, } \\
\text { Manager, Client, Logiciel. }\end{array}$ & $\begin{array}{l}\text { Bon collaborateur: Savoir être, } \\
\text { Personnalité, Relationnel, Humain, } \\
\text { Communication, Écoute, Maturité. } \\
\text { Adaptation: Adaptabilité, Adapté, } \\
\text { Polyvalence. } \\
\text { Inscription dans l'entreprise: Équipe, } \\
\text { Intégration, État d'esprit, Esprit d'équipe, } \\
\text { Valeurs, Connaissance entreprise. }\end{array}$ \\
\hline & \multicolumn{2}{|c|}{$\begin{array}{l}\text { Indicateurs: Salaire, Entretien, Déjà, Adéquation, Intérêt, Test, Correspondance, } \\
\text { Acceptation conditions. }\end{array}$} \\
\hline \multirow[t]{2}{*}{$\begin{array}{l}\text { Capacité à interagir } \\
\text { avec autrui }\end{array}$} & $\begin{array}{l}\text { Compétences commerciales: Commercial, } \\
\text { Vente, Produit. }\end{array}$ & $\begin{array}{l}\text { Bonne présentation: Présentation, } \\
\text { Dynamisme, Sourire, Apparence, Tenue, } \\
\text { Agréable. } \\
\text { Expression: Élocution, Expression, } \\
\text { Langage. } \\
\text { Contact: Contact, Accueil, Amabilité, } \\
\text { Politesse, Enfant, Aisance. } \\
\text { Attitude morale: Propreté, Honnêteté, } \\
\text { Discret, Fiabilité. }\end{array}$ \\
\hline & \multicolumn{2}{|c|}{ Indicateurs: Test situation, Transport, Similaire, Carte professionnelle. } \\
\hline
\end{tabular}

Diplôme, Formation, Niveau ou Profil ${ }^{15}$ ), tantôt des compétences plus pointues comme l'indiquent les termes de Connaissance (dans un domaine précisé), Technique, Langue, Expérience spécialisée, Informatique, Manager. Ce qui est valorisé aussi, c'est l'inscription de la compétence dans une perspective temporelle: les termes de Parcours, Cursus ou Potentiel ouvrent sur l'idée de carrière ou de trajectoire professionnelle. Cette idée se confirme à la lecture des qualités recherchées qui mentionnent l'Adaptabilité du candidat et sa Polyvalence, comme s'il devait être capable d'occuper plusieurs fonctions pour pouvoir évoluer. Davantage qu'un bon travailleur, ce que l'on recherche

15. La mention de Profil donne à penser que le recruteur agit dans une perspective adéquationniste en cherchant à apparier des compétences requises et présentées sur la base de signaux généraux comme la formation ou l'expérience. Cette idée se confirme à la lecture des indicateurs parmi lesquels se trouvent les termes d'Adéquation et de Correspondance. 
en mobilisant ce registre, c'est un bon collaborateur. Dans cette perspective, il doit être doté de Savoir être, d'une Personnalité, d'un bon Relationnel, être capable d'Écoute et de Communication. De telles qualités peuvent être utiles pour faciliter la coopération au sein des entreprises et servir leurs objectifs collectifs. La citation de repères renvoyant au travail en Équipe, à l'Intégration, à la Connaissance de l'entreprise ou à l'adhésion à ses Valeurs corrobore cette idée. La capacité à évoluer dans l'entreprise doit donc être entendue dans son double sens, comme une capacité à s'intégrer dans l'entreprise et à y progresser par la suite.

Du côté des indicateurs, la possibilité d'avoir Déjà travaillé dans l'entreprise donne des assurances au recruteur. Mais d'autres indices sont mobilisés, comme le Salaire demandé ou l'Acceptation des conditions offertes qui laissent entendre que le candidat dispose d'une marge de négociation dans la fixation des conditions d'emploi (ce qui sera confirmé dans la partie 3).

Le dernier registre, celui de la capacité à interagir avec autrui, ne rassemble que 49 repères de sélection. Il est actionné pour recruter des candidats amenés à développer des contacts avec d'autres personnes, comme des clients, des usagers ou un public. Il ne s'agit donc plus de collègues, comme dans le cas précédent où le relationnel est contrôlé pour favoriser la coopération interne. Les repères mobilisés dans ce registre relèvent davantage de qualités que de compétences validées. Seules les compétences commerciales sont mentionnées (Commercial, Produit, Vente), mais celles-ci ne semblent pas résulter du suivi de cursus de formation classique: dans les formulations utilisées par les répondants, il est question de « fibre », « esprit », « posture », « appétence », « sens commercial » ${ }^{16}$. La capacité à interagir avec autrui se décrit davantage à l'aide de qualités professionnelles. Elles s'organisent autour de trois pôles : celui de la présentation de soi (Présentation, Dynamisme, Sourire, Apparence, Tenue, Agréable $)^{17}$, de la capacité à s'exprimer correctement (Élocution, Expression, Langage) et de qualités de contact (Contact, Accueil, Amabilité, Politesse, Aisance). Nous décelons également plusieurs qualités « morales » (Propreté, Honnêteté, Discret, Fiabilité) prisées dans la relation à autrui.

Les quelques indicateurs cités font référence à des tests de situation et aux cartes professionnelles exigées dans le domaine de la sécurité. Mais ils restent peu nombreux. Dans la dernière partie, nous verrons quels sont les modes de recrutement adaptés à l'évaluation de la capacité à interagir avec d'autres ou à se mettre à leur service, dans la mesure où de telles qualités semblent difficilement contrôlables à distance.

\footnotetext{
16. Il s'agit de qualités placées en dehors de toute forme d'apprentissage: si on n'a pas la « sensibilité » ou le « tempérament » ou encore la « fibre », alors « de toute façon on ne fera pas ce métier » (dixit une directrice adjointe en hôtellerie-restauration, citée par BAILLY et al., 2009, p. 48). Et même lorsque les employeurs considèrent que certaines qualités peuvent s'apprendre, ils peuvent faire porter la responsabilité de leur apprentissage sur d'autres acteurs comme l'école ou la famille (BAILLY, LÉNÉ, 2015).

17. Nous retrouvons ici le poids des apparences souligné à propos des métiers d'accueil (HIDRI NEYS, REMICHI, 2015) qui affecte plus largement les emplois supposant des contacts avec clients et usagers
} 
Regardons à présent comment se combinent les trois registres. Puisque le protocole d'enquête veut que les recruteurs puissent mentionner trois critères distincts, ceux-ci appartiennent-ils tous au même registre ou au contraire couvrent-ils plusieurs registres? Comme cela apparaît à la lecture du schéma ci-dessous, la sélection sur le seul registre des capacités à s'investir dans son travail se retrouve dans 20,9\% des cas (classe A), tandis que dans $26,8 \%$ des cas, la sélection est uniquement centrée sur la capacité à évoluer dans l'entreprise (classe B). Ces deux registres disposent donc d'une certaine autonomie, mais leur usage combiné est également fréquent, puisqu'on le retrouve dans près du tiers des cas (classe C, 32,2 \%). Restent toutes sortes de cas de figure, qui représentent au total $19 \%$ des cas, dans lesquels est mentionné le registre des capacités à interagir avec autrui (cas D1, D2, D3 et D4). Celui-ci est mobilisé tantôt seul (cas D1), tantôt conjointement avec un autre (cas D2 et D3) ou les deux autres (cas D4).

Nous faisons le choix de traiter ensemble ces $19 \%$ de cas et de les analyser comme une classe à part entière (la classe D). En effet, le registre des capacités à interagir avec autrui n'a qu'une faible autonomie puisqu'il est très rarement cité seul $(2,2 \%$ des recrutements), mais il n'en présente pas moins des caractéristiques fortes et une certaine homogénéité au regard des types d'emplois à pourvoir dans chaque « cas ${ }^{18}$.

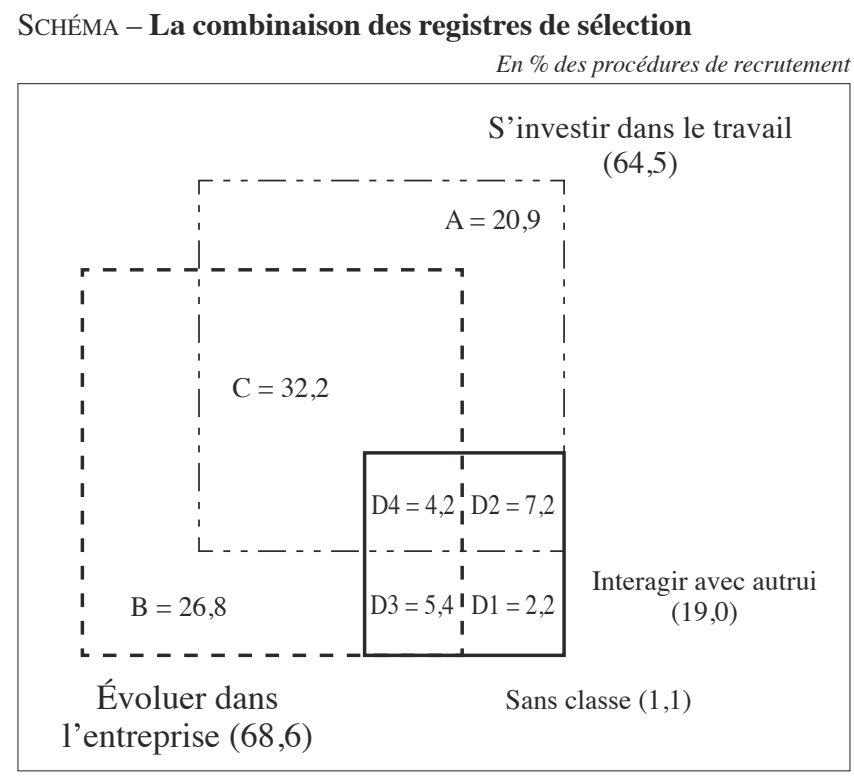

18. Pour ne pas alourdir la présentation, nous renvoyons en annexe 4 le tableau détaillant la répartition des quatre cas (D1, D2, D3 et D4) qui composent la classe D, selon les niveaux de qualification, le temps de travail et le contrat de l'emploi à pourvoir. La proximité entre eux se révèle à la lecture des 10 emplois les plus fréquemment cités dans chaque cas, puisqu'ils concernent souvent des recrutements sur les mêmes emplois. On retrouve ainsi les caissiers de magasins et les vendeurs en alimentation en D1 et D2, les vendeurs en habillement et les assistantes maternelles en D3 et D4, les coiffeurs en D2 et D4, etc. 
Par ailleurs, il se distingue clairement des classes A, B et C au regard de nombreuses caractéristiques, comme nous allons le voir dans la partie suivante. Les quatre classes ainsi dessinées permettent de dresser un portrait assez « équilibré » des procédures de recrutement recensées dans l'enquête, puisqu'elles en représentent de $19 \%$ à $32 \%$.

\section{Un recours différencié selon les emplois, les entreprises et les candidats recrutés}

Ayant décrit le contenu de chacun des registres et la manière dont ils se combinent, nous cherchons maintenant à préciser leurs usages : pour quels types d'emplois sontils mobilisés et par quelles entreprises (tableau 2) ? Pour caractériser les emplois, il semble important de ne pas en rester à l'idée que les qualités et compétences recherchées tiennent uniquement au niveau de qualification du poste à pourvoir, comme cela apparait dans l'AFC. Nous nous interrogeons plus largement ici sur les conditions d'emploi et de travail associées aux registres de sélection, en prenant en compte le titre des emplois à pourvoir, la durée des contrats et le temps de travail. Concernant les entreprises, nous nous intéressons, au-delà des variables classiques (effectifs, activité, appartenance à un groupe), aux moyens dont elles disposent pour aborder le recrutement. Enfin, nous nous attachons à caractériser les profils des candidats recrutés selon que l'un ou l'autre de ces registres est mobilisé, seul ou combiné à d'autres.

TABLEAU 2 - Caractéristiques des emplois et des entreprises selon les registres mobilisés

\begin{tabular}{lccccc} 
& $\begin{array}{c}\text { A: } \\
\text { registre } \\
\text { S'investir } \\
\text { seul }\end{array}$ & $\begin{array}{c}\text { B: } \\
\text { registre } \\
\text { Évoluer } \\
\text { seul }\end{array}$ & $\begin{array}{c}\text { C: } \\
\text { registres } \\
\text { S'investir } \\
\text { et Évoluer }\end{array}$ & $\begin{array}{c}\text { D: } \\
\text { registre } \\
\text { Interagir }\end{array}$ & Ensemble \\
\hline Poids des classes & 20,9 & 26,8 & 32,2 & 19,0 & \\
\hline Qualification du poste & & & & & \\
Ouvrier non qualifié & 29 & 8 & 12 & 9 & 14 \\
Ouvrier qualifié & 20 & 12 & 16 & 11 & 15 \\
Employé non qualifié & 25 & 14 & 22 & 43 & 25 \\
Employé qualifié & 9 & 13 & 13 & 19 & 13 \\
Profession intermédiaire & 13 & 32 & 26 & 15 & 22 \\
Cadre & 4 & 21 & 11 & 3 & 11 \\
& 100 & 100 & 100 & 100 & 100 \\
\hline Type de contrat & & & & & \\
CDI & 43 & 48 & 47 & 50 & 47 \\
CDD de plus de 3 mois & 40 & 39 & 38 & 36 & 38 \\
CDD de 1 à 3 mois & 17 & 13 & 15 & 14 & 15 \\
& 100 & 100 & 100 & 100 & 100 \\
\hline Temps de travail & & & & & \\
Temps plein & 63 & 77 & 70 & 59 & 68 \\
Temps partiel & 37 & 23 & 30 & 41 & 32 \\
& 100 & 100 & 100 & 100 & 100 \\
\hline
\end{tabular}




\begin{tabular}{|c|c|c|c|c|c|}
\hline & $\begin{array}{c}\text { A: } \\
\text { registre } \\
\text { S'investir } \\
\text { seul }\end{array}$ & $\begin{array}{c}\text { B : } \\
\text { registre } \\
\text { Évoluer } \\
\text { seul }\end{array}$ & $\begin{array}{c}\text { C: } \\
\text { registres } \\
\text { S'investir } \\
\text { et Évoluer }\end{array}$ & $\begin{array}{c}\text { D: } \\
\text { registre } \\
\text { Interagir }\end{array}$ & Ensemble \\
\hline \multicolumn{6}{|l|}{ Taille de l'établissement } \\
\hline 1 à 9 salariés & 42 & 27 & 27 & 40 & 33 \\
\hline 10 à 49 salariés & 33 & 32 & 32 & 32 & 32 \\
\hline 50 à 199 salariés & 15 & 19 & 21 & 16 & 18 \\
\hline \multirow[t]{2}{*}{200 salariés et plus } & 10 & 22 & 20 & 12 & 17 \\
\hline & 100 & 100 & 100 & 100 & 100 \\
\hline \multicolumn{6}{|l|}{ Secteur d'activité } \\
\hline Industrie & 11 & 12 & 12 & 9 & 11 \\
\hline Construction & 13 & 9 & 8 & 4 & 9 \\
\hline $\begin{array}{l}\text { Commerce, transports, activités financières } \\
\text { et immobilières }\end{array}$ & 29 & 23 & 23 & 35 & 27 \\
\hline Services aux particuliers & 22 & 11 & 16 & 23 & 17 \\
\hline Services aux entreprises & 13 & 24 & 21 & 15 & 19 \\
\hline \multirow[t]{2}{*}{ Administration, enseignement, santé } & 12 & 21 & 20 & 14 & 17 \\
\hline & 100 & 100 & 100 & 100 & 100 \\
\hline \multicolumn{6}{|l|}{ Appartenance de l'entreprise à un groupe } \\
\hline Non & 69 & 56 & 58 & 63 & 61 \\
\hline \multirow{2}{*}{ Oui } & 31 & 44 & 42 & 37 & 39 \\
\hline & 100 & 100 & 100 & 100 & 100 \\
\hline \multicolumn{6}{|l|}{ Présence d'un département $\mathrm{RH}$} \\
\hline Non & 67 & 45 & 48 & 61 & 54 \\
\hline \multirow[t]{2}{*}{ Oui } & 33 & 55 & 52 & 39 & 46 \\
\hline & 100 & 100 & 100 & 100 & 100 \\
\hline \multicolumn{6}{|l|}{ Existence d'un site Internet } \\
\hline Non & 35 & 20 & 21 & 29 & 25 \\
\hline \multirow[t]{2}{*}{ Oui } & 65 & 80 & 79 & 71 & 75 \\
\hline & 100 & 100 & 100 & 100 & 100 \\
\hline \multicolumn{6}{|l|}{$\begin{array}{l}\text { Nombre de candidatures spontanées } \\
\text { par an }\end{array}$} \\
\hline $\begin{array}{l}\text { Moins que les établissements } \\
\text { de taille similaire }\end{array}$ & 18 & 17 & 15 & 12 & 16 \\
\hline $\begin{array}{l}\text { Autant que les établissements } \\
\text { de taille similaire }\end{array}$ & 55 & 50 & 49 & 50 & 50 \\
\hline \multirow{2}{*}{$\begin{array}{l}\text { Plus que les établissements } \\
\text { de taille similaire }\end{array}$} & 27 & 33 & 36 & 38 & 34 \\
\hline & 100 & 100 & 100 & 100 & 100 \\
\hline
\end{tabular}

Lecture : lorsque le registre des capacités à s'investir dans le travail est mobilisé, $29 \%$ des recrutements concernent des ouvriers non qualifiés.

Champ : les établissements d'au moins un salarié du secteur concurrentiel non agricole (France métropolitaine et DOM) ayant recruté. en CDI ou en CDD de plus d'un mois entre le $1^{\text {er }}$ septembre et le 30 novembre 2015.

Source: Ofer (Dares, 2016); données pondérées.

La capacité à s'investir dans le travail, lorsqu'elle est mobilisée seule (classe A), est particulièrement valorisée dans la sélection des ouvriers non qualifiés (cette classe est composée à $29 \%$ d'ONQ pour $14 \%$ en moyenne sur l'ensemble des classes) et qualifiés. La liste des emplois les plus cités montre qu'il s'agit aussi bien d'apprentis (boulangers, bouchers, charcutiers), de métalliers, serruriers et réparateurs non qualifiés, d'ouvriers non qualifiés du bâtiment (gros œuvre ou second œuvre) et des travaux 
publics, mais aussi de conducteurs livreurs ou de coursiers, d'ouvriers du tri et de nettoyeurs. Donc des métiers où il faut pouvoir se lever tôt, supporter des conditions de travail parfois pénibles ou se déplacer fréquemment. Ces caractéristiques peuvent être rapprochées de l'attention (mentionnée plus haut) portée à la Motivation aussi bien qu'à la Disponibilité ou à la Proximité entre lieu d'habitation et lieu de travail. Les conditions d'emploi ne sont pas nécessairement avantageuses comme le montre la surreprésentation des temps partiels (37\% pour $32 \%$ ) et la sous-représentation des CDI (43\% pour $47 \%$ ) lorsqu'est mobilisé uniquement ce registre. La capacité à s'investir dans le travail est souvent privilégiée dans les établissements de moins de 10 salariés ( $42 \%$ pour $33 \%$ en moyenne), œuvrant dans le domaine des services aux particuliers (22\% pour $17 \%$ ) ou dans celui de la construction (13\% pour $9 \%$ ) où les Savoir-faire sont très prisés. C'est aussi dans ces secteurs d'activité que l'on relève couramment des horaires fragmentés et décalés et des lieux de travail éclatés (BOUFFARTIGUE, 2019). Nous remarquons enfin que les recrutements pour lesquels ce seul registre est mobilisé ont plus souvent lieu dans des entreprises qui ne sont pas dotées de services de ressources humaines ni de sites internet (35\% pour $25 \%$ ). Dans un tel contexte, on suppose que ce sont des opérationnels (le chef d'entreprise ou un supérieur hiérarchique) qui prennent en charge les recrutements et effectuent le contrôle des compétences et qualités recherchées (ce que nous confirmons dans la partie 4).

La capacité à évoluer dans l'entreprise, lorsqu'elle est mobilisée seule (classe B), est utilisée pour pourvoir des emplois très différents des précédents par des entreprises qui le sont également. Il s'agit souvent de recruter des cadres (21\% pour $11 \%$ ) ou des professions intermédiaires (32\% pour $23 \%$ ). Les emplois les plus cités ici concernent les ingénieurs en fabrication ou les chargés d'études et de développement informatique, les animateurs commerciaux, les chefs de chantiers et toutes sortes de cadres. Relativement conformes à la moyenne, les conditions d'emplois offertes s'en distinguent par la surreprésentation des emplois à temps plein (77\% pour $68 \%$ en moyenne). Le registre des capacités à évoluer est davantage mobilisé par de grandes entreprises ( $22 \%$ d'établissements de 200 salariés ou plus), dont les activités sont souvent orientées vers les services aux entreprises (24\% pour $19 \%)$, l'administration, l'enseignement ou la santé ( 21 pour $17 \%$ ). Elles sont bien équipées: elles appartiennent souvent à des groupes, sont fréquemment dotées de départements de ressources humaines (55\% pour $46 \%$ en moyenne) et de sites internet. Plus que la taille ces caractéristiques doivent en fait coïncider avec l'offre de carrières sur des marchés internes, la valorisation du Parcours, de l'Adaptabilité et du Potentiel des candidats.

La mobilisation conjointe des deux registres S'investir et Évoluer (classe C) conduit à une certaine neutralisation des caractéristiques particulières que présente chacun d'eux pris isolément en matière de contrats ou de temps de travail. Elle est caractérisée par une légère surreprésentation des professions intermédiaires (26\% pour $22 \%$ ), occupant des emplois de techniciens et de professionnels, dont les qualifications peuvent être pointues : métreurs et techniciens du bâtiment, plombiers et chauffagistes 
qualifiés, dessinateurs et menuisiers. Mais on y trouve aussi des emplois de services aux personnes (aides à domicile, travailleuses familiales, agents de service hospitaliers, éducateurs spécialisés et assistantes maternelles). Le recours conjoint aux deux registres semble très lié à la taille des entreprises (elles sont plutôt grandes), à leur visibilité sur le marché du travail (étant donné le plus grand volume de candidatures spontanées qu'elles reçoivent et leur site internet). En cela, elles se différencient nettement des entreprises utilisant uniquement le premier registre (qui sont moins souvent dotées d'un site internet et reçoivent plutôt moins de candidatures spontanées) tout en se rapprochant du profil de celles utilisant uniquement le deuxième registre.

Enfin, la capacité à interagir (classe D) est surtout mobilisée dans la sélection des employés qualifiés (19\% pour $13 \%$ ) ou non qualifiés (43\% pour $25 \%$ ). Sans surprise, nous retrouvons ici des emplois de vendeurs et de caissiers, de serveurs et d'agents d'accueil, mais aussi des auxiliaires de puériculture et des manucures. Toutes sortes d'emplois qui ont pour point commun d'être très exposés au regard et aux attentes des clients. Beaucoup de ces emplois s'exercent à temps partiel (41\% pour $32 \%$ en moyenne), mais pour des durées indéterminées (50\% pour $47 \%$ en moyenne). Comme pour le premier registre, ce sont les petits établissements qui privilégient la capacité à interagir (40\% pour $33 \%$ en moyenne), souvent dépourvus de services de ressources humaines (61\% pour $54 \%$ ). Les activités surreprésentées ici sont celles du commerce, du transport et des activités immobilières (35\% pour $27 \%$ en moyenne), ainsi que les services aux particuliers ( $23 \%$ pour $17 \%$ ). Ces secteurs sont emblématiques d'activités où sont valorisées les compétences Commerciales, la Présentation de soi, les qualités d'Expression et de Contact, qu'elles soient ou non associées aux qualités des deux premiers registres.

Le profil des candidats finalement choisis par les recruteurs est également typé selon qu'ils ont privilégié l'un ou l'autre registre de sélection ou leur combinaison, laissant entendre que les caractéristiques sociodémographiques des candidats peuvent guider la sélection qu'effectuent les recruteurs selon les qualités attendues (tableau 3).

Les stéréotypes de genre semblent bien fonctionner pour organiser la division sexuelle du travail d'après des qualités dites masculines ou féminines. Les hommes sont très largement surreprésentés lorsque la capacité à s'investir dans le travail est privilégiée (ils forment $62 \%$ de la classe A pour $53 \%$ en moyenne), c'est-à-dire lorsque sont formulées des attentes en termes d'Aptitude physique, de Savoir-faire ou de Volonté et d'Engagement. Au contraire, ce sont les femmes qui sont plus souvent recrutées lorsque l'accent est mis sur les capacités à interagir avec autrui (56\% de la classe $\mathrm{D}$ pour $47 \%$ en moyenne), là où l'on réclame bonne Présentation, Amabilité, Sourire et Discrétion. Cette division sexuée du travail, qui recouvre aussi une différenciation entre emplois d'ouvriers et d'employés, est probablement entretenue d'un côté par les candidats qui se présentent dans des proportions différentes sur des postes marqués par le genre des personnes les occupant, et de l'autre, par les réflexes discriminatoires des recruteurs qui y puisent des moyens d'accélérer leurs décisions. Ces deux registres de sélection ont en commun d'accuser un déséquilibre en ce qui 
TABLEAU 3 - Caractéristiques des candidats recrutés selon les registres mobilisés

\begin{tabular}{|c|c|c|c|c|c|}
\hline & $\begin{array}{c}\text { A: } \\
\text { registre } \\
\text { S'investir } \\
\text { seul }\end{array}$ & $\begin{array}{c}\text { B : } \\
\text { registre } \\
\text { Évoluer } \\
\text { seul }\end{array}$ & $\begin{array}{c}\text { C: } \\
\text { registres } \\
\text { S'investir } \\
\text { et Évoluer }\end{array}$ & $\begin{array}{c}\mathrm{D}: \\
\text { registre } \\
\text { Interagir }\end{array}$ & Ensemble \\
\hline \multicolumn{6}{|l|}{ Sexe } \\
\hline Femme & 38 & 46 & 49 & 56 & 47 \\
\hline \multirow[t]{2}{*}{ Homme } & 62 & 54 & 51 & 44 & 53 \\
\hline & 100 & 100 & 100 & 100 & 100 \\
\hline \multicolumn{6}{|l|}{ Classe d'âge } \\
\hline Moins de 25 ans & 52 & 35 & 41 & 54 & 44 \\
\hline De 26 à 49 ans & 40 & 57 & 50 & 40 & 48 \\
\hline \multirow[t]{2}{*}{50 ans et plus } & 8 & 8 & 9 & 6 & 8 \\
\hline & 100 & 100 & 100 & 100 & 100 \\
\hline Personne étrangère ou d'origine étrangère & 21 & 15,5 & 16 & 17 & 17 \\
\hline \multicolumn{6}{|l|}{ Diplôme } \\
\hline Ne sait pas & 28 & 22 & 19 & 26 & 23 \\
\hline $\begin{array}{l}\text { Pas de diplôme ou un certificat d'études } \\
\text { primaires, BEPC', brevet des collèges, }\end{array}$ & 21 & 6 & 11 & 12 & 12 \\
\hline $\mathrm{CAP}, \mathrm{BEP}^{*}$ & 17 & 10 & 14 & 11 & 13 \\
\hline $\begin{array}{l}\text { Brevet professionnel, brevet de technicien, } \\
\text { bac professionnel }\end{array}$ & 8 & 8 & 10 & 12 & 9 \\
\hline Bac général, bac technologique & 9 & 5 & 9 & 13 & 9 \\
\hline \multirow{2}{*}{ Supérieur au bac } & 17 & 49 & 37 & 26 & 34 \\
\hline & 100 & 100 & 100 & 100 & 100 \\
\hline
\end{tabular}

\begin{tabular}{|c|c|c|c|c|c|}
\hline \multicolumn{6}{|l|}{ Situation avant l'embauche } \\
\hline Stagiaire & 2 & 3 & 1 & 2 & 2 \\
\hline Intérimaire & 3 & 4 & 3 & 2 & 3 \\
\hline Salarié & 17 & 34 & 26 & 17 & 24 \\
\hline Chômeur & 36 & 34 & 38 & 38 & 36 \\
\hline Étudiant/formation & 23 & 15 & 19 & 24 & 20 \\
\hline \multirow[t]{2}{*}{ Inactif } & 19 & 10 & 13 & 17 & 15 \\
\hline & 100 & 100 & 100 & 100 & 100 \\
\hline $\begin{array}{l}\text { La personne était connue d'un membre } \\
\text { de l'entreprise }\end{array}$ & 38 & 32 & 32 & 27 & 32 \\
\hline $\begin{array}{l}\text { Il y a eu négociation des conditions } \\
\text { d'emploi }\end{array}$ & 7,5 & 16 & 15 & 13 & 13 \\
\hline
\end{tabular}

concerne les classes d'âge: dans les deux cas, ce sont surtout des jeunes (moins de 25 ans) qui sont recrutés ( $52 \%$ ou $54 \%$ de jeunes pour $44 \%$ en moyenne). Ce résultat peut être rapproché d'un autre constat: il s'agit plus souvent également de sortants de formation, d'ex-étudiants ou d'ex-inactifs qui semblent ainsi débuter dans la vie active. Dans les deux cas enfin, on trouve une proportion supérieure à la moyenne de personnes recrutées dont on ne connaît pas le diplôme, indiquant que celui-ci n'a pas de valeur reconnue aux yeux des recruteurs. 
Néanmoins, le registre de la capacité à s'investir dans son travail accuse plusieurs spécificités. Il est davantage marqué que les autres par la présence de non-diplômés ( $21 \%$ pour $12 \%$ ), par celle de personnes d'origine étrangère ( $21 \%$ pour $17 \%$ ) et par des liens entretenus avec des personnes de l'entreprise: en effet, $38 \%$ des candidats sont déjà connus par un de ses membres au moment du recrutement (pour $32 \%$ en moyenne $)^{19}$. Cette introduction ne les met pas pour autant en position de force, puisque les candidats recrutés sur la base de ce registre ont eu moins qu'ailleurs l'opportunité de négocier leurs conditions d'emploi.

À l'opposé, la mobilisation seule du registre des capacités à évoluer dans l'entreprise (classe B) valorise les diplômés, et en particulier ceux du supérieur (49\% pour $34 \%$ en moyenne). Il ne s'agit pas à proprement parler de débutants si l'on en juge par la surreprésentation des candidats déjà salariés au moment de leur embauche et par celle des recrutés de 26 à 49 ans (57\% pour $48 \%)$. Ces caractéristiques laissent entendre que les candidats ne sont pas prêts à accepter n'importe quoi et changent d'emploi dans la perspective de se stabiliser ou d'améliorer leurs conditions d'emploi. Ils sont deux fois plus souvent que les candidats de la classe A en position de négocier.

Pour finir, notons que les profils sélectionnés sur la base conjointe des deux registres $S$ 'investir et Évoluer ne sont pas très marqués au regard des différentes caractéristiques sociodémographiques; les deux registres semblent sur ce point se neutraliser mutuellement.

\section{Les méthodes de recrutement et d'évaluation associées aux registres}

Dans cette dernière partie, nous nous intéressons aux conditions et aux méthodes d'évaluation qui sont associées aux registres de sélection. Il s'agit de mettre en évidence les canaux de recrutement, les ressources mises à disposition, les preuves ( $\mathrm{CV}$, permis, photos, etc.) demandées et les épreuves (entretiens et tests) passées qui doivent concrètement permettre l'évaluation des différents repères privilégiés. Pour ce faire, nous allons procéder à une série de régressions logistiques estimant la sur ou sous-représentation des conditions et méthodes d'évaluation dans chaque classe de recrutements ${ }^{20}$. Comme nous supposons que les entreprises et les emplois structurent

\footnotetext{
19. Notons que lorsque la capacité à interagir est valorisée, ce sont au contraire moins souvent des personnes connues par un membre de l'entreprise qui sont recrutées.

20. Nous procédons à des régressions logistiques dichotomiques testant la probabilité qu'une procédure de recrutement appartienne à telle ou telle classe (A, B, C ou D) plutôt que de ne pas y appartenir. Nous avons écarté la possibilité d'estimer des régressions multinomiales car aucune des quatre classes ne se démarque pour être traitée comme une modalité de référence. De plus, notre stratégie nous permet de conserver les 102 procédures de recrutement qui n'appartiennent à aucun registre.
} 
le choix des critères in fine agrégés dans les registres de sélection, leurs caractéristiques sont systématiquement contrôlées dans l'analyse des résultats ${ }^{21}$.

\section{Les conditions et moyens de recrutement}

Le tableau 4 présente, sous la forme de odds ratio, les relations toutes choses égales par ailleurs entre les quatre classes de recrutements (pour lesquels des critères de registres différents ont été mobilisés) et les conditions de recrutement, à savoir les contraintes (recrutements éventuellement multiples et/ou urgents), les canaux de recrutement précédant la sélection en entreprise et les moyens mis à disposition pour sélectionner les candidatures.

La priorité donnée aux seules capacités à s'investir dans son travail prend place dans le cadre d'entreprises mobilisant peu de moyens humains pour recruter, alors même qu' elles sont soumises à une certaine pression temporelle (classe A, tableau 4). Cela les amène à recourir à leurs réseaux de relations à qui elles délèguent la charge de la sélection. En effet, ces entreprises font plus souvent face à des recrutements urgents, à réaliser en moins d'une semaine, par ailleurs pris en charge par une seule personne, plus vraisemblablement le chef d'entreprise. Rappelons que l'objectif est de s'assurer que l'on a affaire à un bon Travailleur, Sérieux et Assidu, qui connaît son Métier, suffisamment Disponible et prêt à s'Engager dans son travail.

La sélection de candidats est confiée aux réseaux, à celui du personnel en place ou aux réseaux familiaux et amicaux : ils ont une probabilité supérieure à celle de la modalité de référence (les annonces) d'avoir permis de recruter dans cette classe. Les réseaux ont l'avantage de la gratuité, mais pas seulement. Ils permettent d'éviter de formuler des critères formels lorsque ceux-ci n' offrent pas de bons repères pour opérer la sélection et de se reposer sur la personne qui procure la candidature pour contrôler sa qualité (BESSY, MARCHAL, 2009), et fournir les Recommandations et Références qui sont des indicateurs importants dans ce registre où l'on valorise les compétences opérationnelles et le professionnalisme. La surreprésentation de la modalité «moins d'une candidature sur deux écartée » montre aussi que l'action du réseau diminue la charge de la sélection à réaliser en interne. Le canal des écoles et centres de formation, plus fréquent lui aussi lorsque l'on mise sur les capacités de travail, laisse ouverte la possibilité de recruter des débutants, tout en offrant d'autres formes de Références et de garanties via le corps enseignant. Dans l'hôtellerie-restauration (inclus dans le secteur des services aux particuliers surreprésenté dans cette classe), se rapprocher des centres de formation d'apprentis (CFA), écoles ou lycées professionnels permet aussi de remédier aux difficultés de recrutement dans des métiers jugés peu attractifs où il $\mathrm{y}$ a de continuels Besoins de Professionnels disposant de Savoir-Faire (FORTÉ, Monchatre, 2013). Autre canal jouant un rôle privilégié, les candidatures spontanées,

21. C'est particulièrement vrai pour les qualifications à partir desquelles ont été construits les registres. Ce n'est pas le cas des profils recrutés ou des méthodes de recrutement et d'évaluation qui ne structurent pas les classes analysées mais les décrivent. 
TABLEAU 4 - Recours aux registres et conditions de recrutement

\begin{tabular}{|c|c|c|c|c|}
\hline & & & & ipport de chat \\
\hline & $\begin{array}{c}\text { A: } \\
\text { registre } \\
\text { S'investir } \\
\text { seul }\end{array}$ & $\begin{array}{c}\text { B : } \\
\text { registre } \\
\text { Évoluer } \\
\text { seul }\end{array}$ & $\begin{array}{c}\text { C: } \\
\text { registres } \\
\text { S'investir } \\
\text { et Évoluer }\end{array}$ & $\begin{array}{l}\text { D: } \\
\text { registre } \\
\text { Interagir }\end{array}$ \\
\hline $\begin{array}{l}\text { Plusieurs postes identiques à pourvoir } \\
\text { simultanément }\end{array}$ & 1,11 & $0,87 *$ & 0,99 & $1,13^{\bullet}$ \\
\hline $\begin{array}{l}\text { Recrutement urgent (poste à pourvoir } \\
\text { en moins d'une semaine) }\end{array}$ & $1,12^{\bullet}$ & 1,03 & $0,89^{\bullet}$ & 0,99 \\
\hline Canal de recrutement & & & & \\
\hline Réseaux des salariés & $1,25^{\bullet}$ & $0,77 * *$ & 1,07 & 1,04 \\
\hline Réseaux familiaux et amicaux & $1,59 * *$ & $0,62 * *$ & $0,71 *$ & 1,16 \\
\hline Réseaux professionnels & 1,18 & $0,82 *$ & 1,05 & 1,08 \\
\hline Candidatures spontanées de proximité & $1,38 * *$ & $0,63 * * *$ & $0,78 *$ & $1,57 * * *$ \\
\hline Candidatures spontanées à distance & 1,01 & $0,83^{\bullet}$ & 1,09 & 1,14 \\
\hline Annonces (tous supports) & référence & référence & référence & référence \\
\hline CVthèques (entreprise et/ou Internet) & 0,92 & 1,23 & $0,71 *$ & 1,20 \\
\hline $\begin{array}{l}\text { Intermédiaires publics (Pôle emploi, } \\
\text { mission locale et autre service public } \\
\text { de l'emploi) }\end{array}$ & 1,07 & 0,95 & 1,01 & 1,05 \\
\hline Écoles et centres de formation & $1,52 * *$ & $0,75 *$ & 0,97 & 1,13 \\
\hline Intermédiaires privés & 0,82 & 0,99 & 0,96 & $1,33 *$ \\
\hline Rappel de personne ayant déjà travaillé & 0,81 & 1,10 & 1,13 & 0,86 \\
\hline Salon et autres canaux & 0,98 & 1,13 & 1,19 & 0,66 \\
\hline $\begin{array}{l}\text { Intensité du premier tri de candidatures } \\
\text { examinées }\end{array}$ & & & & \\
\hline Moins d'une candidature sur deux écartée & $1,49 * * *$ & $1,13^{\bullet}$ & $0,80 * * *$ & $0,72 * * *$ \\
\hline Une candidature sur deux écartée & référence & référence & référence & référence \\
\hline Plus d'une candidature sur deux écartée & 0,88 & $1,20 * *$ & 0,97 & 0,93 \\
\hline $\begin{array}{l}\text { Nombre de fonctions impliquées } \\
\text { lors de la sélection }\end{array}$ & & & & \\
\hline Une fonction & $1,21 * *$ & $0,86 *$ & 0,94 & 1,03 \\
\hline Deux fonctions & référence & référence & référence & référence \\
\hline Trois fonctions & $0,79 *$ & 1,01 & 1,08 & 1,07 \\
\hline Quatre fonctions et plus & 0,93 & 0,97 & 1,11 & 0,92 \\
\hline $\begin{array}{l}\text { Chef d'entreprise impliqué } \\
\text { dans la sélection }\end{array}$ & $1,20 *$ & $0,86 *$ & 1,00 & 0,97 \\
\hline
\end{tabular}

Notes : régressions logistiques de la probabilité que le recrutement appartienne à une classe donnée plutôt que non.

Modèles avec constante.

$\bullet ; *$;*; *** : significativité respectivement à $10 \% ; 5 \% ; 1 \% ; 0,1 \%$.

Variables de contrôle non mentionnées dans le tableau: la qualification, le type de contrat, le temps de travail, la taille de l'établissement, le secteur d'activité, l'appartenance à un groupe, la présence d'un département RH, l'existence d'un site internet, l'attractivité mesurée par le nombre de candidatures spontanées reçues.

Lecture : un rapport de chances supérieur (inférieur) à 1 indique que la probabilité de mobiliser un registre donné est plus élevée (moins élevée) que pour la catégorie de référence. Par exemple, d'après le premier modèle [registre A], les procédures de recrutement visant à pourvoir un poste en urgence ont un rapport de chances de mobiliser des critères du registre A, plutôt que de ne pas les mobiliser, supérieur d'environ $10 \%(1,12)$ aux procédures de recrutement non urgentes (ayant les mêmes autres caractéristiques).

Champ : les établissements d'au moins un salarié du secteur concurrentiel non agricole (France métropolitaine et DOM) ayant recruté en CDI ou en CDD de plus d'un mois entre le $1^{\text {er }}$ septembre et le 30 novembre 2015.

Source: Ofer (Dares, 2016).

et tout particulièrement les candidatures de proximité. Elles sont déposées par le candidat en personne (RIEUCAU, SALOGNON, 2013), qui peut être soumis à quelques questions rapides à cette occasion et faire l'objet d'une appréciation au jugé (rappelons 
l'importance du Feeling, de l'Attitude, du Respect). Cette entrevue permet également de vérifier la Proximité et la Localisation du candidat, qu'il n'habite pas trop loin du lieu de travail, dispose de moyens de locomotion ou d'un Permis de conduire ${ }^{22}$. Elle donne enfin l'opportunité de s'assurer de sa Disponibilité Immédiate ou de son acceptation de contraintes Horaires. Plus prosaïquement il peut aussi s'agir d'apprécier son Âge ou son Aptitude physique.

La probabilité de recourir uniquement au registre de la capacité à évoluer dans l'entreprise (classe B, tableau 4), qui valorise plus spécifiquement les qualités du bon collaborateur, capable de s'Adapter, de s'Intégrer et de faire Équipe, s'inscrit dans un contexte de recrutement très différent du précédent. Il paraît plus professionnel, ouvert et sélectif, ceci expliquant cela. Il concerne le plus souvent des recrutements uniques, donnant lieu à un tri qui écarte plus d'une candidature sur deux, et impliquant rarement moins de deux fonctions dans l'entreprise. Ainsi, il peut s'agir d'un membre du service des ressources humaines accompagnant un opérationnel, ou bien le responsable de l'unité dans lequel le collaborateur doit être recruté ou un autre responsable. La présence de représentants de différentes fonctions dans l'entreprise agrée la candidature qui acquiert ainsi une légitimité collective. C'est le cas dans les banques commerciales (LARQUIER, TUCHSZIRER, 2013) et les groupes hôteliers (FORTÉ, MONCHATRE, 2013), où les recrutements sont organisés par et avec les « $\mathrm{RH} »$ de sorte que les opérationnels ne recrutent pas uniquement pour eux mais pour l'ensemble de l'enseigne où la carrière va se dérouler. Le chef d'entreprise en revanche a significativement moins de chances d'être impliqué dans la sélection.

Étant donné la valeur des odds ratios significatifs, c'est la modalité de référence, les annonces d'offre d'emploi diffusées sur Internet ou sur un autre support, qui apparaît le canal de recrutement privilégié. La probabilité d'avoir trouvé des candidats via des réseaux, quels qu'ils soient, ou par des candidatures spontanées de proximité est largement plus faible; il en est de même concernant les écoles et centres de formation. En effet, l'importance accordée à l'Expérience est peu compatible avec la recherche de débutants. La valorisation de compétences générales ou spécialisées (entre autres: Diplôme, Formation, Niveau, Cursus, Expérience professionnelle, Technique, Informatique, Logiciel, Langue, Anglais), propres au registre Évoluer, se prête bien à la rédaction d'annonces qui vont permettre de comparer les candidats au regard de ces caractéristiques formelles, comme le laisse penser la présence de repères comme l'Adéquation ou la Correspondance entre profils. Guillemette de LARQUIER et Géraldine RIEUCAU (2017) ont montré que le canal des annonces, très sélectif, amenait à recruter des diplômés du supérieur, pour des postes d'encadrement certes, mais plus encore pour des professions intermédiaires, catégories surreprésentées dans cette classe.

22. La fonction de ces entrevues a été observée dans les salons de recrutement où les interactions entre recruteurs et candidats permettent d'engranger beaucoup d'informations en très peu de temps sur ces sujets (MARCHAL et al., 2017). 
Les conditions d'un recrutement où sont mobilisés conjointement les deux registres S'investir et Évoluer (classe C, tableau 4) ressortent de manière moins évidente que dans les cas où ils sont actionnés seuls. Notons néanmoins que ces recrutements où se cumulent des exigences d'Engagement Immédiat dans l'emploi et de Potentiel à évoluer à plus long terme dans l'entreprise ont peu de chances d'être urgents (à réaliser en moins d'une semaine). Comme lorsque seuls des repères du registre Évoluer sont mobilisés, c'est le canal de recrutement des annonces qui est surreprésenté, mais le premier tri des candidatures est moins intense avec une candidature sur deux écartée.

Les conditions du recours au registre de la capacité à Interagir (classe D, tableau 4) ressortent également peu. L'élément le plus marquant est la place privilégiée des candidatures spontanées de proximité. Nous l'avons dit pour les recrutements liés au registre S'investir, ce mode de candidature permet une première évaluation et des questions rapides, mais il offre surtout l'opportunité de juger en face-à-face la Présentation, l'Apparence, le Langage, la Politesse, la Propreté, etc., du candidat qui s'est déplacé. Enfin, le niveau d'intensité du premier tri est intermédiaire (une candidature sur deux écartée), alors que les recruteurs sont plus souvent dans la nécessité de pourvoir plusieurs postes simultanément ${ }^{23}$.

Nous allons voir que les classes $C$ et $D$ se distinguent davantage en ce qui concerne les éléments demandés (ou non) à l'occasion de la sélection.

\section{Preuves et épreuves de sélection}

Que doivent fournir et à quelles épreuves doivent se soumettre les candidats à l'occasion de la sélection au sein des quatre classes de recrutements ? Nous commentons à présent les résultats des régressions du tableau 5.

Nous avons vu que l'accent mis sur les capacités à s'investir dans le travail passait souvent par des réseaux sollicités en urgence, qui opèrent le filtrage des candidats (LARQUiER, MARChaL, 2012, 2016). Aussi la sélection se résume-t-elle souvent à quelques vérifications: est-ce que le candidat possède bien les certificats voulus, comme des pièces d'identité, des titres de séjour ou des certificats médicaux ? Pour le reste, la mobilisation de ce seul registre (classe A, tableau 5) se caractérise par un moindre recours à des preuves tangibles ou formelles. On y demande moins souvent qu'ailleurs le CV alors qu'il fait partie des repères de ce registre. Une interprétation possible est que le recruteur n'exige pas de CV pour y vérifier des informations, mais valorise les parcours intéressants qualifiés verbalement de «bons $\mathrm{CV}$ » parmi les critères cités, sans que cela ne désigne le document. Ce résultat est confirmé par

\footnotetext{
23. Par ailleurs, on observe un effet surprenant, positif, du canal des intermédiaires privés sur la probabilité qu'une procédure de recrutement appartienne à cette classe qui concerne beaucoup de candidats peu qualifiés. Ce canal (incluant l'Association pour l'emploi des cadres [Apec]) ne représente que $7 \%$ des embauches d'Ofer et concerne surtout des cadres. Qu'il soit surreprésenté dans cette classe révèle peut-être que les intermédiaires privés ont tendance à ajouter le registre Interagir aux registres Évoluer et/ou S'investir, provoquant une inflation des registres (de fait, ils sont surreprésentés dans les cas D3 et D4 présentés en annexe 4).
} 
TABLEAU 5 - Recours aux registres et méthodes de la sélection

\begin{tabular}{|c|c|c|c|c|}
\hline \multicolumn{5}{|c|}{ Rapport de chance } \\
\hline & $\begin{array}{c}\text { A: } \\
\text { registre } \\
\text { S'investir } \\
\text { seul }\end{array}$ & $\begin{array}{c}\text { B : } \\
\text { registre } \\
\text { Évoluer } \\
\text { seul }\end{array}$ & $\begin{array}{c}\text { C: } \\
\text { registres } \\
\text { S'investir } \\
\text { et Évoluer }\end{array}$ & $\begin{array}{c}\mathrm{D}: \\
\text { registre } \\
\text { Interagir }\end{array}$ \\
\hline Demande CV & $0,72 * * *$ & 0,96 & $1,34 * * *$ & $1,43 * *$ \\
\hline Demande lettre motivation & 0,90 & 0,91 & 1,05 & $1,21 * *$ \\
\hline Demande références ou recommandations & $0,86 *$ & 1,08 & $1,13 *$ & 0,92 \\
\hline Demande photo & 1,07 & 0,91 & $0,90^{\bullet}$ & $1,21 * *$ \\
\hline Demande prétentions salariales & $0,79 * * *$ & $1,12^{\bullet}$ & $1,20 * *$ & $0,86 *$ \\
\hline $\begin{array}{l}\text { Demande certificats ou autres documents } \\
\text { administratifs }\end{array}$ & $1,20 * *$ & 0,96 & 0,99 & $0,87 *$ \\
\hline Demande permis de conduire & 1,05 & 0,91 & $1,16 *$ & $0,88^{\bullet}$ \\
\hline $\begin{array}{l}\text { Aucune attente en ce qui concerne le } \\
\text { niveau de diplôme }\end{array}$ & $1,35 * * *$ & $0,74 * * *$ & $0,88 *$ & $1,20 * *$ \\
\hline \multicolumn{5}{|l|}{$\begin{array}{l}\text { Nombre d'entretiens passés } \\
\text { par la personne recrutée }\end{array}$} \\
\hline Au plus un & référence & référence & référence & référence \\
\hline Deux & $0,84 *$ & 1,02 & $1,18 * *$ & 0,94 \\
\hline Trois ou plus & $0,78 *$ & 0,93 & $1,16^{\bullet}$ & 1,03 \\
\hline $\begin{array}{l}\text { Évaluation en situation de travail ou } \\
\text { simulée }\end{array}$ & 1,05 & $0,85 * *$ & 0,96 & $1,31 * * *$ \\
\hline \multicolumn{5}{|l|}{ Autres tests pendant la sélection ${ }^{(a)}$} \\
\hline Aucun & référence & référence & référence & référence \\
\hline Au moins un & $0,74 * * *$ & 1,09 & $1,12^{\bullet}$ & 0,97 \\
\hline
\end{tabular}

Notes : régressions logistiques de la probabilité que le recrutement appartienne à une classe donnée plutôt que non.

Modèles avec constante.

$\bullet ; *$; ; *** : significativité respectivement à $10 \% ; 5 \% ; 1 \% ; 0,1 \%$.

(a) : parmi ceux-ci, des tests de connaissance ou d'intelligence, des tests de personnalité, des tests de langues étrangères (y compris entretiens réalisés dans une langue étrangère), des tests sur les compétences de base (lire, écrire, compter), des épreuves de groupe (plusieurs candidats) ou encore une analyse graphologique.

Variables de contrôle non mentionnées dans le tableau : la qualification, le type de contrat, le temps de travail, la taille de l'établissement, le secteur d'activité, l'appartenance à un groupe, la présence d'un département RH, l'existence d'un site internet, l'attractivité mesurée par le nombre de candidatures spontanées reçues.

Lecture : un rapport de chances supérieur (inférieur) à 1 indique que la probabilité de mobiliser un registre donné est plus élevée (moins élevée) que pour la catégorie de référence. Par exemple, d'après le premier modèle [registre A], les procédures de recrutement demandant aux candidats un $\mathrm{CV}$ ont un rapport de chances de mobiliser des critères du registre A, plutôt que de ne pas les mobiliser, inférieur d'environ $30 \%(0,72)$ aux procédures de recrutement n'exigeant pas de CV (ayant les mêmes autres caractéristiques).

Champ : les établissements d'au moins un salarié du secteur concurrentiel non agricole (France métropolitaine et DOM) ayant recruté en CDI ou en CDD de plus d'un mois entre le $1^{\text {er }}$ septembre et le 30 novembre 2015.

Source: Ofer (Dares, 2016).

l'indifférence au diplôme manifestée par les répondants (modalité Aucune attente à ce sujet). Il est vrai que les parcours scolaires et les diplômes mentionnés dans les CV donnent difficilement à voir des qualités comme la Ponctualité, la Mobilité, la Motivation, la Volonté ou le Sérieux. Les demandes de références ou de recommandations formelles et la soumission à des tests sont également moins fréquentes, puisque les candidats bénéficient de la caution du réseau. De même, on leur demande significativement moins leurs prétentions salariales, comme si le salaire ne posait pas question, fixé indépendamment du candidat et peut-être déjà connu via le réseau. 
Enfin, cette première classe de recrutements se caractérise plus que les autres par un seul entretien, qui est envisagé moins comme une épreuve de sélection que comme un moment d'échange d'informations sur les conditions de travail.

Lorsque l'accent a été mis sur les seules capacités à évoluer, la sélection se distingue par une attente en ce qui concerne le niveau de diplôme tandis qu'une évaluation contextualisée en situation de travail réelle ou simulée est rarement mobilisée (classe B, tableau 5). En effet, les tests de situation n'ont pas de pertinence lorsque le cadre de l'évaluation déborde le poste de travail pour englober plus largement l'entreprise où il faut Évoluer. À cela s'ajoute la demande légèrement plus fréquente de prétentions salariales, en conformité avec la présence des indicateurs Salaire et Acceptation des conditions dans ce registre.

Quand les recruteurs cumulent les deux registres S'investir et Évoluer (classe C, tableau 5), les preuves et épreuves se cumulent également et l'on retrouve alors un certain nombre des indicateurs des deux registres (tableau 1), avec, en premier lieu, la demande du CV. L'attention des recruteurs se porte plus souvent aussi sur les Références et Recommandations ${ }^{24}$, sur les prétentions salariales, c'est-à-dire le Salaire, ainsi que sur le Permis de conduire. À cela s'ajoute une attente sur le niveau de diplôme. Dans la mesure où les compétences nécessaires pour évoluer peuvent être appréciées sur la base du Niveau de Diplôme, celui-ci est considéré comme un repère pertinent. Le candidat doit aussi convaincre ses interlocuteurs et peut passer deux Entretiens, voire trois ou davantage, et avoir au moins un Test à réussir: test de connaissance ou d'intelligence, test de langues ou de personnalité, voire test de Base évaluant la literacy $y^{25}$ (certainement mobilisé quand ce registre est appliqué à des profils moins qualifiés et qu'un diplôme de niveau faible ou inconnu ne garantit pas la qualité des candidats). Le recours aux annonces pour susciter des candidatures (tableau 4), qui a pour corollaire la présentation de candidats inconnus, induit donc ici un renforcement des contrôles, alors que ce n'est pas le cas lorsque seules sont mobilisées les capacités à évoluer (classe B où les annonces sont également plus fréquentes), comme si l'ajout des capacités à s'investir tout de suite nécessitait des preuves plus immédiatement tangibles.

Lorsque le registre Interagir est mobilisé (rappelons-le, éventuellement avec les deux autres registres), les épreuves et preuves se cumulent également lors de la sélection mais de manière différente. Cette fois-ci, ce sont les évaluations en situation de travail ou leur simulation qui sont significativement surreprésentées (classe $\mathrm{D}$, tableau 5). De fait, le repère Test situation est le premier indicateur de ce registre. C'est dans l'action que s'éprouvent le mieux les qualités d'Élocution et d'Expression, les capacités à Vendre, à Accueillir du public ou à établir des Contacts avec des Enfants (BAILLY et al., 2009). L'autre singularité de ce registre tient à la fréquence de la demande

24. Qu'on peut observer pratiquées de manière très organisée, par exemple dans le secteur des services de conseil en informatique (FONDEUR, 2013).

25. Autrement dit les compétences de base à l'écrit (lecture, rédaction, orthographe et grammaire). 
de photographies, qui paraissent utiles pour contrôler l'Apparence, le Sourire ou le look de la personne dont on a souligné l'importance (HIDRI NEYS, REMICHI, 2015; Martin-LaCrouX, LaCrouX, 2017). La demande de photo laisse aussi entendre que les candidats sont souvent des inconnus au moment de la sélection, et que la mise en relation passe par la fourniture d'un CV comportant une photo. De fait, la demande de $\mathrm{CV}$ est forte ici, alors même que ce n'est pas le cursus des candidats qui intéresse les recruteurs, ni leur formation. En témoigne la forte indifférence au diplôme manifestée par les recruteurs dans ce registre où il n'est ni valorisé ni probablement rémunéré. La sous-représentation des prétentions salariales, déjà observée lorsque n'est valorisée que la capacité à s'investir, est également marquée ici. Plus les qualités attendues sont tacites et innées, moins elles sont formalisées, du coup, moins il y a de «barrière à l'entrée » (DevetTer, Puissant, 2018) et de pouvoir de négociation pour les salariés. En revanche, alors que l'on demandait des certificats et autres documents administratifs quand le registre S'investir était mobilisé seul, ces preuves sont ici moins sollicitées; les recruteurs leur préfèrent une lettre de motivation, cette dernière donnant la possibilité d'une première évaluation à distance de l'Expression et du Langage.

Résumons-nous. La valorisation de capacités à s'investir dans son travail (classe A), plus axée sur la recherche de compétences immédiatement opérationnelles, sur la demande de motivation, de disponibilité et de productivité, conduit à miser fortement sur les réseaux et les canaux de proximité pour opérer la sélection. Les réseaux organisent une clôture a priori des recrutements, dans la mesure où ils ne s'adressent pas au tout-venant. Ce faisant, ils permettent d'alléger les épreuves de sélection. Au contraire, la recherche de capacités à évoluer dans les entreprises (classe B), c'est-à-dire de compétences générales ou spécialisées, de qualités axées sur la coopération, l'adaptation et le potentiel, passe par une plus grande ouverture a priori des recrutements, auxquels toutes les parties prenantes semblent pouvoir concourir. Mais la valorisation de ces capacités appelle une forte sélection à distance, basée sur le diplôme. La sélectivité n'est donc pas plus forte dans l'un ou l'autre cas, mais elle ne s'opère pas de la même manière. C'est la mobilisation conjointe des deux registres qui semble donner lieu à des procédures de recrutement plus équipées, si ce n'est plus sélectives (classe $\mathrm{C}$ ). Elles cumulent une sélection à distance, la demande d'un certain nombre de documents et d'informations, des entretiens et des tests pour se donner l'assurance de faire le bon choix, alors que les candidats sont jugés sur deux registres opposés. Enfin, la dernière classe (D) est propre à certaines fonctions où les capacités à interagir sont valorisées à l'interface des entreprises et de leurs clients ou publics. Les recrutements ne sont pas a priori fermés par des réseaux, mais ils privilégient les épreuves en situation et les demandes de documents (CV, lettre de motivation, photo) pour écarter les candidatures inappropriées. 
La diversité des repères de sélection mobilisés à l'occasion des recrutements témoigne de la diversité des préoccupations des employeurs qui, loin de chercher uniquement à recruter des candidats sur la base de compétences balisées par des formations ou des expériences, prennent appui sur toutes sortes de critères pour prendre leurs décisions. Leur variété ne tient pas seulement à celle des formulations adoptées, mais aussi à leur domaine d'application, puisque ces critères concernent aussi bien des qualités personnelles et professionnelles que des conditions d'ordre pratique. Ces caractéristiques posent des défis d'ordre méthodologique affrontés en procédant en plusieurs étapes. La première a consisté à réduire la dispersion du vocabulaire utilisé par les employeurs par diverses opérations de codage et à nous focaliser sur les repères les plus cités, couvrant ainsi $97 \%$ des critères initiaux. En procédant successivement à une $\mathrm{AFC}$ et à une $\mathrm{CAH}$, la seconde étape a permis d'agréger les repères de sélection ordonnés d'après les catégories socioprofessionnelles des postes à pourvoir. Trois registres ont été identifiés: le registre de la capacité à s'investir dans le travail, celui de la capacité à évoluer dans l'entreprise et celui de la capacité à interagir avec autrui. Ces registres de sélection donnent à voir les priorités que se donnent les employeurs. Mais la possibilité de les combiner vient également rappeler que la sélection n'est pas toujours univoque et peut chercher à concilier toutes sortes d'exigences. Les quatre classes de recrutement mises en évidence visent précisément à rendre compte des usages que font les entreprises des registres de sélection et de la manière dont leurs choix sont modulés par leurs propres caractéristiques, celles des postes à pourvoir et des candidats recrutés.

Nos analyses apportent des éclairages importants sur des questions que les approches en termes de compétences comportementales ou de soft skills ont tendance à négliger : à savoir le rôle que jouent les caractéristiques des emplois à pourvoir et des entreprises qui recrutent dans le choix des repères de sélection. Un constat s'impose en effet : la valorisation des repères de sélection est fortement liée aux contextes d'emploi.

Nous montrons d'abord que les repères et les registres de sélection sont différenciés selon les caractéristiques des emplois à pourvoir: selon le niveau de qualification, le temps de travail, les contrats et les types d'emplois. La mise en évidence de tels liens vient ébranler l'idée selon laquelle les compétences dites comportementales, relationnelles ou non cognitives, que nous avons appelées les qualités professionnelles, auraient un caractère transversal ou générique, ou qu'elles seraient nécessaires à l'occupation de toutes sortes d'emplois, indépendamment de leur niveau hiérarchique ou des fonctions à occuper. La recherche de compétences relationnelles, par exemple, souvent considérées comme une condition de l'employabilité, ne recouvre pas les mêmes exigences lorsqu'elle a pour but de s' assurer que le candidat saura faire équipe avec ses collègues (registre des capacités à évoluer) ou lorsqu'elle est destinée à tester sa capacité à faire bonne figure face à des clients mécontents (capacités à interagir). De la même façon, les capacités d'adaptation, mises en exergue dans nombre de référentiels d'emploi, ne sont pas du même ordre lorsqu'elles doivent permettre de changer constamment de lieu de travail (cas du registre des capacités à s'investir), lorsqu'il faut 
s'assurer des possibilités de reconversion de la main-d'œuvre (capacités à évoluer), ou encore de la possibilité de s'ajuster aux desiderata de toutes sortes de bénéficiaires (capacité à interagir). Parce qu'elles ne s'appliquent pas aux mêmes contextes, dans les mêmes entreprises et les mêmes situations de travail, les compétences et qualités ne sauraient être traitées ou évaluées en toute généralité ${ }^{26}$.

Nous avons noté également que les repères sur lesquels les employeurs prennent appui pour sélectionner la main-d'œuvre ne sont pas uniquement des compétences et des qualités professionnelles: la distance au lieu d'habitation, la possession d'un véhicule et d'un permis de conduire, l'apparence et l'âge du candidat, le fait qu'il accepte les conditions offertes jouent un rôle à part entière dans la sélection. L'attention portée à ces éléments, que la littérature passe sous silence, ne s'effectue pas, là encore, in abstracto mais dépend des contextes dans lesquels se déroule le travail, de ses contraintes et avantages. Plusieurs de ces repères flirtent par ailleurs avec des critères discriminatoires et concernent la vie personnelle des candidats dont l'employeur tient compte plus ou moins ouvertement au moment de choisir qui recruter. Leur mention ne manque pas de nous interroger. Ce qui est en cause, ici, c'est l'équilibre des informations dont disposent les deux parties du marché du travail et celle de la répartition du pouvoir de sélection. L'importance primordiale que les employeurs accordent à la Motivation et à la Disponibilité des candidats (rappelons que ces deux repères dépassent l'un et l'autre le millier d'occurrences) corrobore cette idée. Elle semble intervenir tardivement dans le cours du processus (trop tard), au moment du choix final réalisé par l'employeur, alors qu'elle pourrait être du ressort du candidat en amont du recrutement. Si l'on suit ce raisonnement, l'employabilité des individus ne dépend pas seulement de leurs trajectoires ni même de leurs qualités professionnelles, mais aussi de la qualité des informations mises à disposition des candidats par les employeurs. Ces remarques demandent néanmoins à être nuancées en prenant en compte la manière dont s'opèrent les recrutements, comme nous l'avons vu dans la dernière partie.

La mise en évidence du rôle que jouent les modes de recrutement dans la détection des repères de sélection - y compris lorsque sont contrôlés les effets des caractéristiques des entreprises et des postes à pourvoir - constitue un autre apport de cet article. Les repères se prêtent inégalement à la rédaction de profils de compétences requises, tels qu' on peut les lire dans des annonces ou dans des CV. Il s'ensuit qu'ils sont plus ou moins adaptés à la mise en œuvre de recrutements professionnalisés se déroulant en étapes prédéfinies, de la recherche de candidats sur le marché à leur sélection en interne. D'une certaine manière, seul le registre des capacités à évoluer (utilisé seul ou combiné au registre de la capacité à s'investir), particulièrement prisé par les grandes entreprises pour recruter cadres et professions intermédiaires, se prête bien à de telles procédures. Elles permettent de donner la priorité aux diplômes pour se focaliser

26. Nous rejoignons ici le regard que portent les juges sur la validité de ces référentiels (lorsque les entreprises ont à s'expliquer devant les tribunaux sur leurs critères de recrutement), en mettant en doute la possibilité d'évaluer toutes sortes d'emplois à l'aune des mêmes critères et des mêmes exigences, surtout lorsque celles-ci concernent des comportements très généraux. Pour approfondir cette question, on peut se référer à la jurisprudence mentionnée dans la note 7 . 
ensuite sur les qualités professionnelles recherchées. Dès lors que priment les qualités sur les compétences, par exemple la disponibilité sur l'expérience, l'acceptation des conditions de travail ou l'attitude face à un client sur la maîtrise de langages informatiques, d'autres modes de recrutement sont adoptés. L'appui sur les réseaux gagne alors en importance, et plus généralement les canaux de proximité, privilégiés lorsque la capacité à s'investir dans son travail ou à interagir avec autrui est en cause. De la même façon, l'observation des candidats en situation de travail paraît plus pertinente pour apprécier les performances lorsque ces deux registres sont valorisés. Dans les deux cas enfin, les recruteurs précisent qu'ils ne manifestent pas d'attente particulière à l'égard des diplômes.

Cette forme d'indifférence au diplôme du candidat, que manifestent $35 \%$ des recruteurs en moyenne, n'est pas un résultat anodin de l'enquête $O f e r$, ne serait-ce que parce qu'il remet en cause la perspective adéquationniste des politiques publiques focalisées sur l'augmentation des niveaux de formation des populations les moins dotées dans le but de les faire davantage correspondre aux besoins des employeurs. L'analyse de l'éventail des repères de sélection nous invite à prêter attention aux autres attentes des recruteurs, et symétriquement, à mieux prendre en compte la diversité des ressources des demandeurs d'emploi. Cela n'est possible qu'à la condition d'en faire, eux aussi, des acteurs de la sélection.

\section{BIBLIOGRAPHIE}

Aboubadra-Pauly S., Afriat C. (2019), Les Compétences transversales : quels usages sur le marché du travail?, Rapport, Paris, France Stratégie, janvier.

Algan Y., Beasley E., Vitaro F., Tremblay R. (2014), "The Impact of Non-Cognitive Skills Training on Academic and Non-Academic Trajectories: From Childhood to Early Adulthood", décembre.

BaILly F., LÉNÉ A. (2013), "The Personification of the Service Labour Process and the Rise of Soft Skills: A French Case Study", Employee Relations, vol. 35, n 1 1, pp. 79-97.

BAILLY F., LÉNÉ A. (2015), « Post-face: retour sur le concept de compétences non académiques », Formation emploi, n ${ }^{\circ} 130$, pp. 69-78.

BaILly F., LÉNÉ A., Toutin M.-H. (2009), «La portée formatrice de l'expérience: le cas des employeurs du secteur des services », Formation emploi, $\mathrm{n}^{\circ} 106$, pp. 41-58.

BELLIER S. (1998), Le Savoir-Être dans l'entreprise. Utilité en gestion des ressources humaines, Paris, Vuibert.

Bensidoun I., Trancart D. (2018), « Choix professionnels et écarts de salaires entre hommes et femmes: le rôle des différences de préférences et d'attitudes face au travail », Population, vol. 73, n 1, pp. 35-62.

BeRgeAt M., RÉMY V. (2017), «Comment les employeurs recrutent-ils leurs salariés? », Dares analyses, $\mathrm{n}^{\circ} 064$. 
Bessy C., Eymard-Duvernay F., LARQuier G. De, Marchal E. (dir.) (2001), Des marchés du travail équitables? Une approche comparative France Royaume-Uni, Bruxelles, Peter Lang - Presses interuniversitaires européennes.

Bessy C., Marchal E. (2009), «Le rôle des réseaux et du marché dans les recrutements », Revue française de socio-économie, $\mathrm{n}^{\mathrm{O}} 3$, pp. 121-146.

Bouffartigue P. (2019), « Temporalités : les nouvelles formes de disponibilité au travail », in Mercure D., Vultur M. (dir.), Dix concepts pour penser le nouveau monde du travail, Paris/ Québec, Hermann/Presses de l’Université Laval, pp. 209-227.

Boussard-Verrecchia E., Petrachi X. (2012), « Système d'évaluation et critères comportementaux : vers une clarification (À propos de CA Toulouse 21 septembre 2011, Airbus) », Le Droit ouvrier, $\mathrm{n}^{\circ} 762$, pp. 1-6.

Callaghan G., Thompson P. (2002), "We Recruit Attitude: The Selection and Shaping of Routine Call Center Labour”, Journal of Management Studies, vol. 39, n 2, pp. 233-254.

CAPPElli P. (1995), “Is the 'Skills Gap' Really about Attitudes?”, California Management Review, vol. 37, $\mathrm{n}^{\circ} 4$, pp. 108-124.

Chambonnaud L. (1918), La Technique des affaires (méthodes françaises et étrangères). III. Les Affaires et le personnel, Paris, Dunod.

Collard D., Suquet J.-B., Raulet-Croset N. (2015), « La gestion paradoxale des compétences de service », Formation emploi, n ${ }^{\circ} 130$, pp. 49-67.

Demazière D., Marchal E. (2018), « La fabrication du travail non qualifié. Analyser les obstacles à la valorisation », Travail et Emploi, $\mathrm{n}^{\mathrm{o}}$ 155-156, pp. 5-30.

DÉPRET E., FilisETti L. (2001), « Juger et estimer la valeur d'autrui : des biais de jugement aux compétences sociales », L'Orientation scolaire et professionnelle [en ligne], n ${ }^{\circ}$ 30/3. https:// doi.org/10.4000/osp.5090

Devetter F.-X., Puissant E. (2018), « Mécanismes économiques expliquant les bas salaires dans les services à la personne. Une analyse centrée sur les aides à domicile », Travail et Emploi, n ${ }^{\circ} 155-156$, pp. 31-64.

Duru-Bellat M. (2015), «Les compétences non académiques en question », Formation emploi, $\mathrm{n}^{\circ} 130$, pp. 13-29.

ÉDUCATION PERM ANENTE (2019a), «Quelle reconnaissance des compétences transversales? » (dossier), $\mathrm{n}^{\circ} 218$.

ÉDUCATION PERM ANENTE (2019b), « Partir des compétences transversales pour lire autrement le travail (Afpa 2019)» (dossier), $n^{\circ}$ HS 13.

EYMARD-DUVERNAY F., MARCHAL E. (1997), Façons de recruter : le jugement des compétences sur le marché du travail, Paris, Métailié.

EYMARD-Duvernay F., MARCHAL E. (2000), « Qui calcule trop finit par déraisonner : les experts du marché du travail », Sociologie du travail, vol. 42, n 3, p. 411-432.

FONDEUR Y. (2013), «Services de conseil en informatique: recruter pour placer », La Revue de l'Ires, $\mathrm{n}^{\mathrm{o}} 76$, p. 99-125. 
FORM ATION EM PLOI (2015), « L'essor des compétences non académiques » (dossier), $\mathrm{n}^{\circ} 130$.

Forté M., Monchatre S. (2013), « Recruter dans l'hôtellerie-restauration : quelle sélectivité sur un marché du travail en tension? », La Revue de l'Ires, n $^{\circ}$ 76, pp. 127-150.

FRANCE STRATÉGIE (2017), Compétences transférables et transversales. Quels outils de repérage, de reconnaissance et de valorisation pour les individus et les entreprises?, Rapport du groupe de travail $n^{\circ} 2$ du réseau Emplois Compétences, Paris, France Stratégie, avril.

Grugulis I., Vincent S. (2009), "Whose Skill is it Anyway? 'Soft' Skills and Polarization”, Work, Employment and Society, vol. 23, n ${ }^{\circ}$, pp. 597-615.

Heckman J. J., Kautz T. (2012), “Hard Evidence on Soft Skills”, Labour Economics, vol. 19, $\mathrm{n}^{\mathrm{o}} 4$, pp. 451-464.

Heckman J. J., Stixrud J., Urzua S. (2006), “The Effects of Cognitive and Noncognitive Abilities on Labor Market Outcomes and Social Behavior", Journal of Labor Economics, vol. $24, \mathrm{n}^{\circ} 3$, pp. 411-82.

Hidri Neys O., Remichi Y. (2015), «"Privé versus Public”. Le poids des apparences dans le recrutement du personnel d'accueil », La Nouvelle Revue du travail [en ligne], $\mathrm{n}^{\circ} 7 . \mathrm{https}^{\prime / /}$ doi.org/10.4000/nrt.2398

JANY-CATRICE F. (2007), «La dévalorisation des services "relationnels" dans les pratiques et les conventions dominantes », L'Homme et la société, n ${ }^{\circ}$ 163-164, pp. 15-34.

Kautz T., Heckman J. J., Diris R., Ter Weel B., Borghans L. (2014), Fostering and Measuring Skills Improving Cognitive and Non-cognitive Skills to Promote Lifetime Success, Paris, OECD.

Kracauer S. ([1929] 2004), Les Employés. Aperçus de l'Allemagne nouvelle, trad. Claude Orsoni, Paris, Éditions de la MSH.

LAINÉ F. (2016), « Les compétences attendues par les employeurs et les pratiques de recrutement », Éclairages et Synthèses, n ${ }^{\circ} 22$, Pôle emploi.

LAINÉ F. (2018), Situations de travail, compétences transversales et mobilité entre les métiers, Note de synthèse, Paris, France Stratégie, février.

LARQUIER G. DE (2016), Une approche conventionnaliste du marché du travail fondée sur le recrutement des entreprises, Mémoire en vue de l'obtention de l'habilitation à diriger des recherches, Université Paris Ouest Nanterre la Défense.

LARQuier G. DE, Marchal E. (2012), « La légitimité des épreuves de sélection : apports d'une enquête statistique auprès des entreprises », in Eymard-Duvernay F. (dir.), Épreuves d'évaluation et chômage, Toulouse, Octarès Éditions, pp. 47-77.

Larquier G. DE, Marchal E. (2016), "Does the Formalization of Practices Enhance Equal Hiring Opportunities? An Analysis of a French Nation-Wide Employer Survey", SocioEconomic Review, vol. 14, n 3 , pp. 567-589.

LARQUIER G. DE, RIEUCAU G. (2017), « Les annonces d'offre d'emploi : une information publique mais ciblée. Exploitation de l'enquête Emploi (2003-2012) », Revue économique, vol. 68, n² pp. 199-217. 
LARQuier G. DE, Rieucau G. (2019), "Valuation and Matching: A Conventionalist Explanation of Labour Markets by Firm's Recruitment Channels”, Historical Social Research, vol. 44, $\mathrm{n}^{\mathrm{o}} 1$, pp. 52-72.

LARQUIER G. DE, TUCHSZIRER C. (2013), « Le secteur bancaire: des recrutements sous l'autorité des ressources humaines? », La Revue de l'Ires, n 76, pp. 71-98.

LEDUC S., VALLÉRY G. (2006), «Approche des compétences sociales par l'analyse de l'activité : une étude chez les aides à domicile », L'Orientation scolaire et professionnelle, $\mathrm{n}^{\circ} 35 / 3$, pp. 387-419.

Marchal E. (2005), « Graphologie et entreprises: histoire et controverses », Sociologies pratiques, $\mathrm{n}^{\mathrm{o}} 10$, pp. $57-76$.

Marchal E. (2015), Les Embarras des recruteurs. Enquête sur le marché du travail, Paris, Éditions de l'École des hautes études en sciences sociales.

Marchal E., Remillon D., Rieucau G. (2017), «Éprouver le marché du travail dans les salons de l'emploi », Socio-économie du travail, n 2, pp. 101-129.

Martin-Lacroux C., Lacroux A. (2017), « Souriez, vous êtes recruté... ? : l'effet des indices et signaux dans les choix de présélection des recruteurs », $28^{e}$ Congrès de l'AGRH, Aix-enProvence, octobre.

Merle V. (1993), «La refonte du ROME: une approche renouvelée de la réalité des métiers », Grand Angle sur l'emploi (ANPE), nº 6, pp. 9-38.

Mills C. W. (1970), Les Cols blancs. Essai sur les classes moyennes américaines, trad. A. Chassigneux, Paris, François Maspero.

Moss P., Tilly C. (1996), “Soft Skills and Race: An Investigation of Black Men's Employment Problems", Work and Occupations, vol. 23, n 3, pp. 252-276.

Pôle EMPLOI (2018), «La nécessaire construction d'un référentiel partagé de compétences transversales - Entretien avec Frédéric Lainé » [en ligne] http://www.emploiparlonsnet. pole-emploi.org/articles/savoir-manipuler-des-petits-objets-est-une-competence-ou-de-lanecessite-de-construire-un-referentiel-partage-de-competences-transversales-entretien-avecfrederic-laine/, consulté le 6 décembre 2021.

RIEUCAU G. (2015), “Getting a Low-Paid Job in French and UK Supermarkets: From Walk-in to Online Application?”, Employee Relations, vol. 37, n 1 1, pp. 141-156.

Rieucau G., SALOGNON M. (2013), « Le recrutement dans la grande distribution: des pratiques ajustées? », La Revue de l'Ires, nº 76, pp. 45-69.

Warhurst C., NicKson D. (2007), "Employee Experience of Aesthetic Labour in Retail and Hospitality”, Work Employment and Society, vol 21, n 1, pp. 103-120.

Zamudio M., Lichter M. (2008), "Bad Attitudes and Good Soldiers: Soft Skills as a Code for Tractability in the Hiring of Immigrant Latina/os over Native Blacks in the Hotel Industry", Social Problems, vol. 55, nº 4, pp. 573-589. 


\section{ANNEXeS}

ANNEXE 1 - Les 34 premières lignes du tableau de contingence qui en compte 224 (encadré 3 ) correspondant aux repères de sélection ayant au moins 100 occurrences

\begin{tabular}{|c|c|c|c|c|c|c|}
\hline & Cadre & PI & ENQ & EQ & ONQ & OQ \\
\hline EXPERIENCE & 230 & 501 & 485 & 232 & 235 & 300 \\
\hline MOTIVATION & 105 & 305 & 448 & 197 & 380 & 266 \\
\hline COMPETENCE & 275 & 443 & 247 & 211 & 156 & 243 \\
\hline DISPONIBILITE & 90 & 262 & 414 & 124 & 223 & 141 \\
\hline PRESENTATION & 10 & 67 & 189 & 97 & 64 & 67 \\
\hline DIPLOME & 52 & 194 & 80 & 84 & 17 & 42 \\
\hline SAVOIR-ETRE & 46 & 107 & 117 & 49 & 54 & 57 \\
\hline PERSONNALITE & 99 & 150 & 60 & 58 & 18 & 41 \\
\hline CONNAISSANCE & 47 & 86 & 49 & 51 & 55 & 42 \\
\hline SERIEUX & 10 & 59 & 77 & 42 & 71 & 69 \\
\hline TECHNIQUE & 95 & 112 & 20 & 23 & 24 & 43 \\
\hline PONCTUALITE & 4 & 15 & 93 & 19 & 68 & 57 \\
\hline EXP PROFESSIONNELLE & 23 & 69 & 46 & 29 & 29 & 33 \\
\hline RELATIONNEL & 34 & 68 & 59 & 28 & 16 & 16 \\
\hline PROXIMITE & 8 & 34 & 72 & 20 & 47 & 38 \\
\hline DYNAMISME & 10 & 32 & 99 & 23 & 30 & 17 \\
\hline FORMATION & 34 & 62 & 22 & 28 & 22 & 23 \\
\hline METIER & 24 & 36 & 22 & 17 & 26 & 47 \\
\hline RIGUEUR & 8 & 37 & 37 & 26 & 26 & 22 \\
\hline NIVEAU & 22 & 65 & 15 & 21 & 13 & 11 \\
\hline SALAIRE & 33 & 52 & 6 & 13 & 7 & 28 \\
\hline ENVIE & 6 & 17 & 43 & 7 & 39 & 21 \\
\hline PERMIS & 2 & 9 & 22 & 5 & 32 & 61 \\
\hline ENTRETIEN & 17 & 32 & 27 & 26 & 14 & 12 \\
\hline SAVOIR FAIRE & 8 & 21 & 34 & 9 & 21 & 35 \\
\hline ADAPTABILITE & 19 & 38 & 22 & 21 & 17 & 10 \\
\hline DEJA & 16 & 31 & 19 & 16 & 22 & 21 \\
\hline LOCALISATION & 2 & 22 & 33 & 9 & 22 & 35 \\
\hline FEELING & 8 & 29 & 36 & 18 & 14 & 16 \\
\hline RECOMMANDATION & 17 & 22 & 17 & 14 & 27 & 19 \\
\hline CONNAISSANCE PERSONNELLE & 8 & 24 & 19 & 19 & 19 & 24 \\
\hline ADEQUATION & 36 & 37 & 15 & 14 & 6 & 2 \\
\hline COMPORTEMENT & 12 & 17 & 23 & 14 & 20 & 22 \\
\hline AUTONOMIE & 13 & 25 & 15 & 7 & 16 & 31 \\
\hline
\end{tabular}

Note $: \mathrm{PI}=$ profession intermédiaire $; \mathrm{ENQ}=$ employé non qualifié $; \mathrm{EQ}=$ employé qualifié $; \mathrm{ONQ}=$ ouvrier non qualifié $; \mathrm{OQ}=$ ouvrier qualifié. 
ANNEXE 2 - Contributions, $\cos ^{2}$ et coordonnées des 15 premières lignes (repères de sélection) et des 6 colonnes (qualifications) du tableau de contingence sur les 2 premiers axes de l'AFC

Les lignes et les colonnes sont classées par ordre décroissant de leur contribution à la construction de l'axe (ce sont celles qui expliquent le plus les variations des observations). Le $\cos ^{2}$ indique la qualité de représentation sur chaque axe, représentation donnée par les coordonnées.

\begin{tabular}{lcrr}
\hline \multicolumn{4}{c}{ Les 15 repères contribuant le plus à l'axe 1 } \\
\hline Repères & $\begin{array}{c}\text { Contribution } \\
\text { par ordre } \\
\text { décroissant }\end{array}$ & Cos $^{2}$ & $\begin{array}{c}\text { Coord. } \\
\text { sur l'axe } \\
\text { 1 }\end{array}$ \\
\hline TECHNIQUE & 7,480 & 0,821 & 0,510 \\
COMPETENCE & 6,961 & 0,906 & 0,221 \\
PERSONNALITE & 6,779 & 0,971 & 0,419 \\
PONCTUALITE & 4,469 & 0,915 & $-0,439$ \\
MOTIVATION & 4,358 & 0,772 & $-0,168$ \\
DIPLOME & 3,779 & 0,613 & 0,298 \\
ADEQUATION & 2,991 & 0,792 & 0,548 \\
SALAIRE & 2,660 & 0,812 & 0,460 \\
PRESENTATION & 2,561 & 0,509 & $-0,239$ \\
SOURIRE & 2,087 & 0,336 & $-0,485$ \\
MANAGER & 2,003 & 0,620 & 0,940 \\
DISPONIBILITE & 1,971 & 0,542 & $-0,132$ \\
NIVEAU & 1,845 & 0,782 & 0,372 \\
PERMIS & 1,613 & 0,265 & $-0,369$ \\
POTENTIEL & 1,547 & 0,792 & 0,709 \\
FORMATION & 1,388 & 0,918 & 0,283 \\
\hline
\end{tabular}

\begin{tabular}{lcrr}
\hline \multicolumn{3}{c}{ Les 15 repères contribuant le plus à l'axe 2 } \\
\hline Repères & $\begin{array}{c}\text { Contribution } \\
\text { par ordre } \\
\text { décroissant }\end{array}$ & Cos $^{2}$ & $\begin{array}{c}\text { Coord. } \\
\text { sur l'axe } \\
\mathbf{2}\end{array}$ \\
\hline SOURIRE & 6,551 & 0,534 & $-0,611$ \\
PERMIS & 6,261 & 0,520 & 0,516 \\
ACCUEIL & 5,073 & 0,759 & $-0,698$ \\
CONDUITE & 3,435 & 0,308 & 0,858 \\
PRESENTATION & 3,426 & 0,344 & $-0,197$ \\
AMABILITE & 2,934 & 0,583 & $-0,584$ \\
VENTE & 2,701 & 0,733 & $-0,697$ \\
APTITUDE & 2,670 & 0,426 & 0,588 \\
PHYSIQUE & 2,624 & 0,465 & $-0,263$ \\
DYNAMISME & 2,087 & 0,736 & 0,260 \\
METIER & 1,849 & 0,620 & 0,381 \\
ASSIDUITE & 1,788 & 0,919 & $-0,619$ \\
AISANCE & 1,689 & 0,680 & $-0,318$ \\
ELOCUTION & 1,656 & 0,696 & $-0,351$ \\
EXPRESSION & 1,589 & 0,442 & 0,477 \\
BESOIN & 1,575 & 0,129 & $-0,137$ \\
DIPLOME & & & \\
\hline
\end{tabular}

\begin{tabular}{|c|c|c|c|c|c|c|c|}
\hline \multicolumn{4}{|c|}{ Les qualifications sur l'axe 1} & \multicolumn{4}{|c|}{ Les qualifications sur l'axe 2} \\
\hline Qualifications & $\begin{array}{c}\text { Contribution } \\
\text { par ordre } \\
\text { décroissant }\end{array}$ & $\operatorname{Cos}^{2}$ & $\begin{array}{c}\text { Coord. } \\
\text { sur l'axe } \\
\quad 1\end{array}$ & Qualifications & $\begin{array}{l}\text { Contribution } \\
\text { par ordre } \\
\text { décroissant }\end{array}$ & $\operatorname{Cos}^{2}$ & $\begin{array}{c}\text { Coord. } \\
\text { sur l'axe } \\
\quad 2\end{array}$ \\
\hline Cadre & 0,762 & 34,867 & 0,642 & ENQ & 0,438 & 37,292 & $-0,306$ \\
\hline PI & 0,713 & 23,222 & 0,348 & OQ & 0,497 & 29,753 & 0,351 \\
\hline ENQ & 0,487 & 20,968 & $-0,323$ & ONQ & 0,300 & 23,549 & 0,315 \\
\hline ONQ & 0,440 & 17,463 & $-0,382$ & EQ & 0,217 & 7,476 & $-0,189$ \\
\hline OQ & 0,093 & 2,824 & $-0,152$ & Cadre & 0,016 & 1,438 & 0,093 \\
\hline $\mathrm{EQ}$ & 0,038 & 0,657 & 0,079 & PI & 0,008 & 0,493 & $-0,036$ \\
\hline
\end{tabular}


ANNEXE 3 - Composition exhaustive des trois registres de sélection (nombre d'occurrences entre parenthèses)

Registre 1 - «S'investir dans le travail »

( 92 repères de sélection: $64,5 \%$ des recrutements)

MOTIVATION (1701), DISPONIBILITE (1 254), SERIEUX (328), PONCTUALITE (256), PROXIMITE (219), METIER (172), RIGUEUR (156), ENVIE (133), PERMIS (131), SAVOIR-FAIRE (128), LOCALISATION (123), FEELING (121), RECOMMANDATION (116), CONNAISSANCE PERSONNELLE (113), COMPORTEMENT (108), AUTONOMIE (107), QUALIFICATION (97), VOLONTE (94), BASE (87), PROFESSIONNALISME (82), HORAIRE (75), MOBILITE (73), AGE (72), AUCUN (72), ASSIDUITE (71), CONFIANCE (65), ATTITUDE (64), CV (63), PROJET (63), REACTIVITE (61), REFERENCE (60), RAPIDITE (58), APTITUDE (55), CAPACITE (55), PROFESSIONNELLE (55), GEOGRAPHIQUE (52), RESPECT (49), ORGANISATION (48), IMMEDIAT (46), APTITUDE PHYSIQUE (43), ESSAI (42), BESOIN (39), COMPREHENSION (36), EFFICACITE (36), TRAVAILLEUR (35), FRANÇAIS (31), APPRENDRE (29), ELIGIBLE (29), COURAGE (28), QUALITE TRAVAIL (28), CONDUITE (26), INTELLIGENCE (25), QUALITE (25), CARACTERE (23), BIEN FAIRE (22), ENGAGEMENT (22), IMPLICATION (21), JEUNE (21), SOCIABILITE (18), APPRENTISSAGE (17), PAS DE CHOIX (17), RESPONSABILITE (17), SCOLAIRE (16), STABILITE (16), FAMILLE (15), FLEXIBILITE (15), INITIATIVE (15), CONNAISSANCE GEO (12), AMBITION (11), CUISINE (11), TRAVAIL (11), BULLETIN (10), SECURITE (10), SITUATION (10), TRAVAIL GROUPE (10), DETERMINATION (9), QUALITE PERSONNELLE (9), CERTIFICAT (8), IMPOSE (8), NETTOYAGE (8), PASSION (8), PERIODE ESSAI (8), SAVOIR TRAVAILLER (8), SPONTANEITE (8), MINUTIE (7), NOTES (7), APPLICATION (6), ATTENTIF (6), CONSCIENCIEUX (6), OPERATIONNEL (6), PRODUCTIVITE (6), STATUT (6)

\section{Registre 2 - «Évoluer dans l'entreprise»}

( 83 repères de sélection: $68,6 \%$ de recrutements)

EXPERIENCE (1 983), COMPETENCE (1 575), DIPLOME (469), SAVOIR-ETRE (430), PERSONNALITE (426), CONNAISSANCE (330), TECHNIQUE (317), EXPERIENCE PROFESSIONNELLE (229), RELATIONNEL (221), FORMATION (191), NIVEAU (147), SALAIRE (139), ENTRETIEN (128), ADAPTABILITE (127), DEJA (125), ADEQUATION (110), PROFIL (96), LANGUE (88), EQUIPE (83), INTERET (71), PARCOURS (70), INTEGRATION (68), EXPERIENCE SPE (66), ANGLAIS (63), TEST (62), HUMAIN (56), ADAPTE (54), COMMUNICATION (48), ETAT ESPRIT (47), ESPRIT EQUIPE (46), POLYVALENCE (45), INFORMATIQUE (44), CORRESPONDANCE (40), POTENTIEL (34), ECOUTE (30), COMPETENCE SPE (29), MATURITE (29), ACCEPTATION CONDITIONS (27), MANAGER (25), CLIENT (22), CURSUS (21), LOGICIEL (21), ANCIENNETE (20), EMPATHIE (19), VALEURS (19), ADHESION (18), CONNAISSANCE ENTREPRISE (17), ALTERNANCE (16), PEDAGOGIE (16), PERFORMANCE (16), PUBLIC (16), DUREE (14), 
INVESTISSEMENT (14), MAITRISE (14), OUVERTURE (14), COHERENCE (13), ECOLE (13), ENSEIGNEMENT (12), EXPERTISE (12), MOBILITE GEO (12), ANALYSE (11), EVOLUTION (11), SAVOIR VIVRE (11), VISION METIER (11), ANIMATION (10), BAFA (10), HANDICAP (10), BAC (9), CONNAISSANCE ETABLISSEMENT (9), ETUDES (9), LOYAUTE (9), DEPLACEMENT (8), LA PERSONNE (8), REDACTION (8), REPUTATION (8), TEMPS PARTIEL (8), CREATIVITE (7), CURIOSITE (7), ADOLESCENT (6), ARGUMENTATION (6), DOMAINE (6), ENTHOUSIASME (6), SENS SERVICE (6)

\section{Registre 3 - « Interagir avec autrui »}

(49 repères de sélection: $19 \%$ des recrutements)

PRESENTATION (494), DYNAMISME (211), SOURIRE (98), ELOCUTION (93), EXPRESSION (75), COMMERCIAL (73), CONTACT (70), ACCUEIL (58), AMABILITE (48), PROPRETE (42), HONNETETE (41), POLITESSE (41), TEST SITUATION (35), ENFANT (34), TRANSPORT (34), DISCRET (32), FIABILITE (31), VENTE (31), AISANCE (26), TENUE (25), PRODUIT (21), SIMILAIRE (20), APPARENCE (19), CARTE PROFESSIONNELLE (18), AGREABLE (16), ETUDIANT (16), LANGAGE (16), GENTILLESSE (14), HYGIENE (13), PATIENCE (13), SYMPATHIE (13), PRESENCE (12), SPORTIF (11), ACCEPTATION (10), BON SENS (10), CHARISME (10), PRESTANCE (9), SINCERITE (9), EDUCATION (8), AUTORITE (7), URGENCE (7), AVENANT (6), DEBROUILLARD (6), EXPERIENCE METIER (6), LETTRE MOTIVATION (6), PARLER (6), SOIN (6), TEMPERAMENT (6), VIVACITE (6) 
ANNEXE 4 - Caractéristiques des « sous-cas » du registre Interagir avec autrui

\begin{tabular}{|c|c|c|c|c|c|}
\hline & $\begin{array}{c}\text { D1: } \\
\text { registre } \\
\text { Interagir } \\
\text { seul }\end{array}$ & $\begin{array}{c}\text { D2: } \\
\text { registres } \\
\text { Interagir } \\
\text { et } S \text { 'investir }\end{array}$ & $\begin{array}{c}\text { D3 : } \\
\text { registres } \\
\text { Interagir } \\
\text { et Évoluer }\end{array}$ & $\begin{array}{c}\text { D4: } \\
\text { registres } \\
\text { Interagir, } \\
\text { S'investir } \\
\text { et Évoluer }\end{array}$ & $\begin{array}{c}\text { Ensemble } \\
\text { de la classe D }\end{array}$ \\
\hline Poids de la classe & 2,2 & 7,2 & 5,4 & 4,2 & 19,0 \\
\hline \multicolumn{6}{|l|}{ Qualification du poste } \\
\hline Ouvrier non qualifié & 7 & 13 & 8 & 7 & 10 \\
\hline Ouvrier qualifié & 5 & 15 & 7 & 12 & 11 \\
\hline Employé non qualifié & 58 & 50 & 30 & 40 & 43 \\
\hline Employé qualifié & 16 & 13 & 27 & 18 & 19 \\
\hline Profession intermédiaire & 11 & 7 & 23 & 18 & 15 \\
\hline \multirow[t]{2}{*}{ Cadre } & 3 & 1 & 5 & 5 & 3 \\
\hline & 100 & 100 & 100 & 100 & 100 \\
\hline \multicolumn{6}{|l|}{ Temps de travail } \\
\hline Temps plein & 60 & 55 & 63 & 62 & 60 \\
\hline \multirow[t]{2}{*}{ Temps partiel } & 40 & 45 & 37 & 38 & 41 \\
\hline & 100 & 100 & 100 & 100 & 100 \\
\hline \multicolumn{6}{|l|}{ Type de contrat } \\
\hline CDI & 52 & 52 & 46 & 48 & 50 \\
\hline CDD de plus de 3 mois & 32 & 34 & 40 & 36 & 36 \\
\hline \multirow[t]{2}{*}{ CDD de 1 à 3 mois } & 15 & 14 & 14 & 15 & 14 \\
\hline & 100 & 100 & 100 & 100 & 100 \\
\hline
\end{tabular}

Champ : les établissements d'au moins un salarié du secteur concurrentiel non agricole (France métropolitaine et DOM) ayant recruté en CDI ou en CDD de plus d'un mois entre le $1^{\text {er }}$ septembre et le 30 novembre 2015.

Source : Ofer (Dares, 2016); données pondérées. 
